\title{
Superior immunity that allows neutralization of all SARS-CoV-2 variants of concern develops in COVID- 19 convalescents and naïve individuals after three vaccinations
}

\section{Ulrike Protzer ( $\sim$ protzer@tum.de )}

School of Medicine, Technical University of Munich / Helmholtz Zentrum München

https://orcid.org/0000-0002-9421-1911

\section{Paul Wratil}

LMU Munich

\section{Marcel Stern}

Max von Pettenkofer Institute \& Gene Center, Virology, LMU München https://orcid.org/0000-00027692-6905

\section{Alina Priller}

Institute of Molecular Immunology and Experimental Oncology, University Hospital rechts der Isar,

Technical University of Munich (TUM), School of Medicine

\section{Annika Willmann}

Institute of Virology, Helmholtz Center Munich / Technical University of Munich (TUM), School of

Medicine

\section{Giovanni Almanzar}

Department of Pediatrics, Pediatric Rheumatology/Special Immunology, University Hospital Wuerzburg, Josef-Schneider-Str. 2,

\section{Emanuel Vogel}

Institute of Virology, Helmholtz Center Munich / Technical University of Munich (TUM), School of Medicine https://orcid.org/0000-0001-6560-0136

\section{Martin Feuerherd}

Institute of Virology,Technical University / Helmholtz Center Munich https://orcid.org/0000-0002-52726503

\section{Cho-Chin Cheng}

Institute of Virology, Helmholtz Center Munich / Technical University of Munich (TUM), School of Medicine

\section{Sarah Yazici}

Institute of Molecular Immunology and Experimental Oncology, University Hospital rechts der Isar, Technical University of Munich (TUM), School of Medicine

\section{Catharina Christa}


Institute of Virology, Helmholtz Center Munich / Technical University of Munich (TUM), School of Medicine

\section{Samuel Jeske}

Institute of Virology, Helmholtz Center Munich / Technical University of Munich (TUM), School of Medicine

\section{Gaia Lupoli}

Max-von-Pettenkofer Institute and Gene Center, Virology, Faculty of Medicine, LMU München

\section{Manuel Albanese}

Max-von-Pettenkofer Institute and Gene Center, Virology, Faculty of Medicine, LMU München

\section{Ernesto Mejías-Pérez}

Max-von-Pettenkofer Institute and Gene Center, Virology, Faculty of Medicine, LMU München

\section{Stefan Bauernfeind}

Gene Center, LMU München

\section{Natalia Graf}

Institute of Virology, Helmholtz Center Munich / Technical University of Munich (TUM), School of Medicine

\section{Hrvoje Mijocevic}

Institute of Virology, Helmholtz Center Munich / Technical University of Munich (TUM), School of Medicine

\section{Martin Vu}

Institute of Virology, Helmholtz Center Munich / Technical University of Munich (TUM), School of Medicine

\section{Kathrin Tinnefeld}

Institute of Virology, Helmholtz Center Munich / Technical University of Munich (TUM), School of Medicine

\section{Jochen Wettengel}

Institute of Virology, Helmholtz Center Munich / Technical University of Munich (TUM), School of Medicine

\section{Dieter Hoffmann}

Institute of Virology, Helmholtz Center Munich / Technical University of Munich (TUM), School of Medicine

\section{Maximilian Muenchhoff}

Pettenkofer Institute https://orcid.org/0000-0001-7016-0470

\section{Christopher Dächert}

Max-von-Pettenkofer Institute and Gene Center, Virology, Faculty of Medicine, LMU München https://orcid.org/0000-0001-6441-3099

\section{Helga Mairhofer}

Max-von-Pettenkofer Institute and Gene Center, Virology, Faculty of Medicine, LMU München

\section{Stefan Krebs}


Ludwig Maximilian University of Munich

\section{Philipp Steininger}

Institute of Clinical and Molecular Virology, University Hospital Erlangen, Friedrich-Alexander Universität Erlangen-Nürnberg,

\section{Helmut Blum}

Laboratory for Functional Genome Analysis, Gene Center, Ludwig Maximilians University Munich https://orcid.org/0000-0002-3994-2332

\section{Veit Hornung}

Ludwig-Maximilians-Universität https://orcid.org/0000-0002-4150-194X

\section{Bernhard Liebl}

Bavarian Health and Food Safety Authority (LGL)

\section{Klaus Überla}

University Hospital Erlangen, Friedrich-Alexander University Erlangen-Nürnberg

\section{Martina Prelog}

Department of Pediatrics, Pediatric Rheumatology/Special Immunology, University Hospital Wuerzburg

\section{Percy Knolle}

Technical University of Munich https://orcid.org/0000-0003-2983-0414

\section{Oliver Keppler}

Ludwig Maximilians University Munich https://orcid.org/0000-0002-1384-8946

\section{Volker Fingerle}

Bavarian Health and Food Safety Authority (LGL)

\section{Alexander Graf \\ University of Munich}

\section{Article}

Keywords: SARS-CoV-2, COVID-19, variant of concern, omicron, neutralizing antibodies, vaccination, immunity, breakthrough infection

Posted Date: January 6th, 2022

DOI: https://doi.org/10.21203/rs.3.rs-1226339/v1

License: (c) (1) This work is licensed under a Creative Commons Attribution 4.0 International License. Read Full License

Version of Record: A version of this preprint was published at Nature Medicine on January 28th, 2022. See the published version at https://doi.org/10.1038/s41591-022-01715-4. 
[Research Article]

\section{Superior immunity that allows neutralization of all SARS-CoV-2 variants of concern develops in COVID-19 convalescents and naïve individuals after three vaccinations}

Paul R. Wratil ${ }^{1,2 \dagger}$, Marcel Stern ${ }^{1 \dagger}$, Alina Priller ${ }^{3 \dagger}$, Annika Willmann ${ }^{4}$, Giovanni Almanzar ${ }^{5}$, Emanuel Vogel ${ }^{4}$, Martin Feuerherd ${ }^{4}$, Cho-Chin Cheng ${ }^{4}$, Sarah Yazici ${ }^{3}$, Catharina Christa ${ }^{4}$, Samuel Jeske ${ }^{4}$, Gaia Lupoli ${ }^{1}$, Tim Vogt ${ }^{5}$, Manuel Albanese ${ }^{1, \#,}$, Ernesto Mejías-Pérez ${ }^{1}$, Stefan Bauernfried $^{6}$, Natalia Graf ${ }^{4}$, Hrvoje Mijocevic ${ }^{4}$, Martin $\mathrm{Vu}^{4}$, Kathrin Tinnefeld ${ }^{4}$, Jochen Wettengel $^{2,4}$, Dieter Hoffmann ${ }^{2,4}$, Maximilian Muenchhoff ${ }^{1,2}$, Christopher Daechert ${ }^{1}$, Helga Mairhofer ${ }^{1}$, Stefan $\mathrm{Krebs}^{7}$, Volker Fingerle ${ }^{8}$, Alexander Graf ${ }^{7}$, Philipp Steininger ${ }^{9}$, Helmut Blum $^{7}$, Veit Hornung ${ }^{6}$, Bernhard Liebl ${ }^{8}$, Klaus Ueberla ${ }^{9}$, Martina Prelog ${ }^{5}$, Percy Knolle ${ }^{3^{*}}$, Oliver T. Keppler ${ }^{1,2^{*}}$, Ulrike Protzer ${ }^{2,4^{*}}$

${ }^{1}$ Max von Pettenkofer Institute and Gene Center, Virology, National Reference Center for Retroviruses, Faculty of Medicine, LMU München, Munich, Germany

${ }^{2}$ German Centre for Infection Research (DZIF), Partner Site, Munich, Germany

${ }^{3}$ Institute of Molecular Immunology and Experimental Oncology, University Hospital rechts der Isar, Technical University of Munich (TUM), School of Medicine, Munich, Germany

${ }^{4}$ Institute of Virology, Helmholtz Center Munich / Technical University of Munich (TUM), School of Medicine, Munich, Germany

${ }^{5}$ Pediatric Rheumatology / Special Immunology, Pediatrics Department, University Hospital Würzburg, Würzburg, Germany.

${ }^{6}$ Gene Center and Department of Biochemistry, LMU München, Munich, Germany

${ }^{7}$ Laboratory for Functional Genome Analysis, Gene Center, LMU München, Munich, Germany

${ }^{8}$ Bavarian Health and Food Safety Authority (LGL (LGL), Oberschleißheim, Germany

9 Institute of Clinical and Molecular Virology, University Hospital Erlangen, Friedrich-Alexander Universität Erlangen-Nürnberg, Erlangen, Germany

$\dagger$ These authors contributed equally to this work

* These authors equally share responsibility for this work

\#Present address: National Institute of Molecular Genetics (INGM), Milano, Italy 


\title{
${ }^{*}$ Corresponding authors:
}

Oliver T. Keppler, M.D., Max von Pettenkofer Institute, Virology, LMU München, FeodorLynen-Straße 23, 81377 Munich, Germany. Phone: +49 (0)89 - 218072901, E-mail: keppler@mvp.uni-muenchen.de

Ulrike Protzer, M.D., Institute of Virology, Technical University of Munich / Helmholtz Zentrum München, Trogerstr. 30, 81675 Munich, Germany. Phone: +49-(0)89-41406886, E-mail: protzer@tum.de; protzer@helmholtz-muenchen.de

\begin{abstract}
Infection-neutralizing antibody responses after SARS-CoV-2 infection or COVID-19 vaccination are an essential part of antiviral immunity. This immune protection is challenged by the occurrence of SARS-CoV-2 variants of concern (VoCs) with immune escape properties, such as omicron (B.1.1.529) that is rapidly spreading worldwide. Here, we report neutralizing antibody dynamics in a longitudinal cohort of COVID-19 convalescent and naïve individuals vaccinated with mRNA BNT162b2 by quantifying anti-SARS-CoV-2-spike antibodies and determining their avidity and neutralization capacity. A superior infection-neutralizing capacity against all VoCs, including omicron, developed by either two vaccinations of convalescents, or a third vaccination or breakthrough infection of twice-vaccinated naïve individuals. These three consecutive spike antigen exposures resulted in an increasing neutralization capacity per antispike antibody unit and were paralleled by stepwise increases in antibody avidity. In conclusion, an infection/vaccination-induced hybrid immunity or a triple immunization induces high-quality antibodies resulting in superior neutralization capacity against VoCs, including omicron.
\end{abstract}

\section{Keywords}

SARS-CoV-2; COVID-19; variant of concern; omicron; neutralizing antibodies; vaccination; immunity; breakthrough infection 


\section{Main Text}

The World Health Organization classified B.1.1.529 on November 26, 2021 as a SARS-CoV2 variant of concern (VoC), named omicron. Already in the last weeks of December 2021, omicron has become the dominant $\mathrm{VoC}$ in many countries, including Great Britain, France, Denmark, Greece, Portugal and the US ${ }^{1}$. Earlier VoCs showed either an enhanced ability for transmission (VoCs alpha (B.1.1.7) and delta (B.1.617.2)) or a partial immune escape with variable effects on neutralization by polyclonal serum antibodies (VoCs beta (B.1.351), gamma (P.1/B.1.1.28)) and delta) $)^{2-7}$. A striking characteristic of the apparently independently evolved VoC omicron is the large number of amino acid substitutions, insertions and deletions in the spike protein, i.e. 32 compared to the original Wuhan-hu- 1 virus $^{8}$, that likely contribute to its extraordinarily rapid spread in the population. Since the number of epitopes in the spike protein relevant for neutralization and targeted by polyclonal antibody responses in COVID-19 convalescent or vaccinated naïve individuals is an important determinant of the genetic barrier to viral escape from humoral immunity ${ }^{6,9}$, physician-scientists have been alerted early on by omicron's potential for a pronounced immune escape

Neutralizing antibody levels are highly predictive of immune protection from symptomatic SARS-CoV-2 infection ${ }^{10}$. Affinity maturation of neutralizing antibodies can markedly alter their capacity to control SARS-CoV-2 variants ${ }^{11}$. In general, somatic hypermutations in variable regions of antibodies increase their binding affinity depending on type and duration of antigen exposure ${ }^{6,12}$. Affinity maturation can markedly expand the breadth and efficiency of neutralizing antibodies against SARS-CoV- $2^{13}$. This may even enable the neutralization of emerging virus variants that have evolved to escape neutralization by ancestral antibodies.

In this study, we characterized the antibody response in a longitudinal cohort of 98 convalescent individuals, infected with SARS-CoV-2 during the first pandemic wave in spring 2020, and 73 naïve individuals matched for sex, age, working conditions and risk factors (Koerber et al. Nature Communications, 2022 in press). These two groups were monitored after the first, second and third vaccination with the mRNA BNT162b2 COVID-19 vaccine. Serum samples were characterized for anti-spike IgG titers, IgG antibody avidity and infectionneutralizing capacity. To quantify infection neutralization, we employed a novel, highthroughput virus neutralization assay with all known VoCs isolated from COVID-19 patients. Hereby, immortalized human MDA-MB-231 cells expressing the angiotensin-converting 
enzyme 2 (hACE2) receptor (MDA-MB-231-hACE2 cells) ${ }^{14,15}$, which are highly susceptible to SARS-CoV-2 infection and display a strong cytopathic response, allowed for the rapid quantification of neutralizing activities against SARS-CoV-2.

Using this assay, we demonstrated that COVID-19 convalescents show a more sustained neutralization capacity after one vaccination with mRNA BNT162b2 than naïve individuals vaccinated twice. A third vaccination was required in naïve individuals to develop the high antibody avidity needed for an improved protection against VoCs with potential for humoral immune escape. Moreover, our results indicate that a breakthrough infection of vaccinees with either $\mathrm{VoC}$ delta or omicron can be as effective as a third vaccine shot. Thus, three timely spaced events of spike antigen exposure can induce superior neutralizing immunity essential to combat a $\mathrm{VoC}$ such as omicron with an unprecedented ability of immune escape.

Taken together, our findings indicate that a superior serum infection-neutralizing capacity against SARS-CoV-2 and its VoCs, including omicron, can develop from "hybrid immunity" based on different combinations of vaccination and infection in COVID-19 convalescents and naïve individuals. Hereby, a high infection-neutralization capacity developed in serum over time following a total of three spike antigen exposures and was paralleled by an increasing avidity of SARS-CoV-2 spike-binding lgG antibodies, providing a critical refinement of the assessment of antibody titers.

\section{Following COVID-19 vaccination, convalescents develop a higher infection- neutralization capacity against all SARS-CoV-2 VoCs than naïve individuals}

(for details see, Extended Data Fig. 1, and Koerber et al., Nature Communications 2022 in press). These individuals were continuously followed since the first wave of the COVID-19 pandemic in spring 2020, through their initial COVID-19 vaccinations with mRNA BNT162b2 in early 2021 and after a third vaccination during the last quarter of 2021 with a total of 486 serum samples collected. In this cohort, we determined the dynamics of anti-SARS-CoV-2 spike antibodies and serum neutralization capacity against the early clinical SARS-CoV-2 isolate B.1.177 (EU1) and all five VoCs: B.1.1.7 (alpha), B.1.351 (beta), P.1/B.1.1.28.1 (gamma), B.1617.2 (delta) as well as B.1.1.529 (omicron) (Extended Data Fig. 1). The first 
(\#1) and second (\#2) COVID-19 vaccination were given three weeks apart, and the third vaccination (\#3) was applied 9 months later.

Sera from COVID-19 convalescents collected approx. 9 months after infection showed a low-level infection-neutralization capacity against the early 2020 SARS-CoV-2 variant EU1 as well as all VoCs (Fig. 1a). After a first vaccination with mRNA BNT162b2, serum neutralization titers of convalescents showed a 63-fold increase on average, while titers in naïve vaccinees remained close to background (Fig. 1b). Neutralization titers in naïve individuals markedly increased after vaccination \#2, still remaining significantly lower than those of convalescents (Fig. 1c). Interestingly, even at 4 and 7 months after vaccination \#2, no significant difference in neutralization capacity was detected comparing convalescents vaccinated once or twice within a three-week interval (Fig. 1d, Extended Data Fig. 2). Although in naïve individuals the infection-neutralization capacity after vaccination \#2 did not reach levels comparable to convalescents (Fig. 1a-d), the relative ability of individual VoCs to escape neutralization relative to EU1 at 7 months after vaccination \#2 was similar for convalescent and naïve individuals (Fig. 1e, Extended Data Fig. 3). Overall, the infectionneutralization capacity for omicron and, albeit less pronounced, for beta was lower than for the other SARS-CoV-2 variants confirming the immune escape properties of these particular VoCs (Fig. 1a-e, Extended Data 2,3). 40.6\% (95\% confidence interval: $29.4-52.9$ \%) of naïve individuals, but only $4.0 \%$ (95\% confidence interval: $1.1-13.5 \%$ ) of convalescents showed no neutralization activity against omicron 7 months after the initial vaccinations.

Strikingly, after COVID-19 vaccination \#3 administered 9 months after vaccinations \#1 and \#2, the infection-neutralization capacity against all VoCs, including omicron, reached high levels in both naïve and convalescent individuals (Fig. 1f). Again, infection-neutralization capacity remained higher in vaccinated convalescents, and there was no difference whether convalescents had received vaccination \#2 or not (Fig. 1f). Fig. 1g summarizes neutralization of VoCs compared to that of EU1, highlighting both the prominent immune escape properties of omicron and the impact of a third vaccination in naïve individuals that was able to partially counteract this pathogen evolution.

Overall, COVID-19 convalescents showed a significantly higher neutralization capacity against all SARS-CoV-2 variants compared to naïve individuals, even after three vaccinations. The omicron $\mathrm{VoC}$ is characterized by an unprecedented escape from antibody neutralization 95 in serum samples from convalescents and naïve individuals at all time-points of this study. 
The increased infection-neutralization capacity is associated with higher antibody avidity

The higher neutralization capacity of convalescents in light of the immune escape properties of the omicron $\mathrm{VoC}$ prompted us to investigate the longitudinal dynamics of infectionneutralization and compare these to antibody titers and avidity. Serum anti-spike IgG levels reached their maximum in convalescents already after vaccination \#1, in naïve individuals after vaccinations \#2 (Fig. 2a). Subsequently, IgG levels declined in both groups at 4 months and even more so at 7 months after vaccination \#2, albeit more rapidly in naïve individuals (Fig. 2a). After vaccination \#3, serum anti-spike IgG levels increased drastically compared to 7 months after the initial vaccinations, on average by a factor of 2.7 and 9.6 for vaccinated convalescent and naïve individuals, respectively, (Fig. 2a).

The marked decline in serum anti-spike IgG levels in both study groups following vaccination \#2 (Fig. 2a) was contrasted by a rather stable infection-neutralization capacity against all VoCs (Extended Data Fig. 4) including delta and omicron (Fig. 2b,c). In naïve individuals, neutralization capacity that was particularly low against omicron significantly increased after vaccination \#3 (Fig. 2b,c). In convalescents, vaccination \#3 further increased their capacity to neutralize EU1 as well as alpha, gamma and omicron, but not beta or delta VoCs (Fig. 2b,c, Extended Data Fig. 4, 5). Specifically, the neutralization capacity against delta, reflected by the $\mathrm{IC}_{50}$ value, showed an 8.1 -fold increase in naïve individuals, but only a 4.6-fold increase in convalescents (Fig. 2d). Against omicron, $>42$-fold increase in naïve individuals and a $>14$-fold increase in convalescents, respectively, were observed (Fig. 2e), indicating the particular relevance of a third vaccination to be able to neutralize the omicron VoC.

To better assess the relative efficacy of serum antibodies for virus neutralization we determined the ratio between the $\mathrm{IC}_{50}$ neutralization and anti-spike $\lg \mathrm{G}$ titers. Notably, we observed a high neutralization capacity per antibody unit in sera of convalescents against EU1 and all VoCs, including omicron, that slightly increased after vaccination \#2 and more pronounced after vaccination \#3 (Fig. 2f,g, Extended Data Fig. 6). For naïve individuals, in contrast, this ratio was low after vaccination \#1 and \#2, increased over time ( $\mathrm{m} 4$ and $\mathrm{m} 7$ ) and further after vaccination \#3 reaching levels comparable to those seen in convalescents (Fig. 2f,g, Extended Data Fig. 6).

Collectively, these results indicate an antibody maturation over time and after each encounter with the SARS-CoV-2 spike protein. Conceptually, this could be due to either an 

CoV-2 breakthrough infections corroborated the findings from the longitudinal analysis in the 
first cohort: Both for vaccinated naïve and for convalescent individuals a total of three challenges of the immune system with SARS-CoV-2 spike antigen, irrespective of the type of exposure, i.e. vaccination or infection, led to superior infection-neutralization capacity.

\section{Discussion}

Using a rapid and sensitive high-throughput infection-neutralization assay with clinical isolates of all known SARS-CoV-2 VoCs we quantified and compared the serum neutralization capacity in a longitudinal cohort of COVID-19 convalescents and matched naïve individuals and determined its distinct dynamics. Comparison to a second cohort of vaccinated individuals with recent delta and omicron breakthrough infections allowed defining the common endpoint of a superior neutralization capacity against all SARS-CoV-2 VoCs, including the emergent omicron VoC.

175 We here report four key findings: First, in a direct comparison with all other VoCs, omicron displays the most pronounced humoral immune escape evading antibody neutralization at early and late time points after vaccination. Second, a "hybrid immunity" in convalescents after one mRNA vaccination is not further enhanced by a second vaccination after a short time frame of three weeks. In contrast, a timely spaced, second vaccination after several months further increases neutralization capacity of most VoCs, especially omicron. Third, in a longitudinal analysis there is no strong correlation between anti-spike IgG titers and the infection-neutralization capacity. A stepwise increase in the avidity of SARS-CoV-2 spikespecific antibodies after the first vaccination in convalescents and after the second and third vaccination in naïve individuals was noted, consistent with the reported occurrence of hypermutated memory $B$ cells up to 6 months after infection ${ }^{17}$, highlighting that the quality rather than the mere quantity of antibodies is important. Forth, triple-vaccinated naïve individuals reach almost the same level of neutralization capacity against the immune escape VoC omicron as vaccinated convalescents. Thus, the more rapid induction of high-avidity antibodies in convalescents and subsequent vaccination can be compensated for by three mRNA vaccinations in naïve individuals.

"Hybrid immunity" was achieved either after two mRNA vaccinations in convalescents (first cohort) or after a SARS-CoV-2 breakthrough infection in naïve individuals, who had received a complete COVID-19 vaccination cycle (second cohort) resulting in superior neutralizing immune responses. An alternative path towards a comparably high neutralizing 
195 similar observations by others ${ }^{18,19}$.

From our data we conclude that a high infection-neutralization capacity of SARS-CoV2 VoCs - including those with immune escape properties- needs to develop over time following a total of three spike antigen exposures. These dynamics were paralleled by an increasing assessment of antibody titers in predicting the efficacy of protective humoral responses against VoCs.

\section{Methods}

\section{Study participants and sample collection}

In a screening effort, 4554 health care workers were tested for SARS-CoV-2 nucleocapsidspecific antibodies with a commercial chemiluminescence immunoassay (iFlash CLIA, YHLO Biotechnology, China) (Erber et al, accepted for publication 2022). Convalescents from SARSCoV-2 infection in the first pandemic wave in March/April 2020 were identified either by positive PCR or by two to four independent serological assays (specificity of $\geq 98 \%$ for each assay results in a specificity of $\geq 99.96 \%$ for the convalescent cohort) (Koerber et al, Nature Communications 2022, in press). Naïve individuals tested negative in at least two different SARS-CoV-2 nucleocapsid-specific IgG assays. 171 (98 convalescent and 73 naïve) individuals were enrolled into a follow-up study that was conducted from April 2020 onwards at the University Hospital rechts der Isar of the Technical University of Munich. Studies were approved by the local ethics committee (ethics vote 476/20 and 26/21S-SR) and participants gave written informed consent to study participation and biobanking.

68 convalescents gave written informed consent for further analyses after their COVID-19 vaccination. 73 SARS-CoV-2 naïve individuals were matched by sex, age, working conditions and risk factors present in the convalescent cohort. Six convalescent individuals were excluded because they showed $a \geq 4$ - and $\geq 8$-fold increase in a surrogate neutralization and in $\mathrm{IC}_{50}$ neutralization, respectively, independent of vaccination indicating SARS-CoV-2 re-exposure (Koerber et al, Nature Communications 2022, in press). Median age was 36 (interquartile range [IQR] 29 to 53) years in naïve and 38 (IQR 29 to 53) years in convalescent participants. 65.8\% naïve and $54.1 \%$ convalescent participants were female. All naïve and 25/68 convalescent individuals continuously followed-up received two doses of BNT162b2 mRNA-vaccine 
(Comirnaty ${ }^{\mathrm{TM}}$, Biontech/Pfizer) as immunization. The interval between the two vaccinations was on average 22 and 21 days for naïve and convalescent individuals, respectively. Due to a change in the national guidelines in March 2021 remaining 43/68 convalescents from the first wave were only vaccinated once with BNT162b2 until mid 2021 assuming that the prior infection substitutes for one vaccination ${ }^{20}$.

Sera from 34 naïve and 51 convalescent participants were analyzed prior to vaccination, from 48 naïve and 59 convalescent participants two weeks after the first vaccination and from 47 naïve and 23 convalescent participants two weeks after the second vaccination. 45 and 72 naïve and 51 and 56 convalescent participants were tested four and seven months after their basic immunization, respectively, including 31 and 37 of convalescents who did not receive a second vaccine dose. Finally, sera from 59 naïve participants and 36 convalescents were evaluated 2 weeks after receiving an additional BNT162b shot as third immunization after on average 9 months (Extended Data Fig. 1).

Additionally, a second cohort of 29 individuals with PCR-confirmed breakthrough infections with SARS-CoV-2 delta or omicron $\mathrm{VoC} \geq 14$ days after completed immunization were included (CoVaKo cohort, Extended Data Table 2). This study was approved by the local ethics committee (ethics-vote 229/21) and all participants gave written informed consent. Median age was 35 (IQR 31 to 38) years in delta- and 42 (IQR 28 to 52) years in omicroninfected participants. Specimens were collected on average 8 days after the first positive PCR result proving breakthrough infection. VoC-specific PCR and/or whole genome sequencing identified delta (B.1.617.2) in respiratory samples of 16/29 and omicron (B.1.1.529) in in respiratory samples of $13 / 29$ individuals. In this cohort, 24/29 participants (83\%) had received two doses of an mRNA vaccine (20 BNT162b2, 4 mRNA-1273). 5/29 had received a first vaccination with an adenoviral vector vaccine, two of which subsequently received the same vaccine and two were vaccinated with BNT162b2 (suppl. Table 1). Median time span between first positive PCR result and a complete vaccination cycle was 141 (IQR 99 to 242) days in delta-infected and 172 (IQR 156 to 207) days in omicron-infected individuals.

\section{Antibody detection and avidity assays}

IgG-type antibody responses to the S1 domain of SARS-CoV-2 spike antigen were quantified in 10-fold diluted serum specimens using the commercial Anti-SARS-CoV-2 QuantiVac-ELISA (lgG) (Eurolmmun, Germany). Binding strength of the SARS-Cov-2 IgG antibodies was 
determined by adaptation of the commercial IgG agile SARS-CoV-2 ELISA (Virion/Serion, Germany) using ammonium thiocyanate (NH4SCN) (Roth, Germany) as chaotropic agent as described previously ${ }^{21}$. Briefly, serum samples were measured using the IgG agile SARS-CoV2 ELISA and adjusted to $100 \mathrm{BAU} / \mathrm{mL}$ according to the standard curve provided by the manufacturer to exclude an influence of variable antibody concentrations. Then, serum samples were incubated in plates pre-coated with SARS-CoV-2-spike-ectodomain S1, S2 and $\mathrm{RBD}$ recombinant antigens (Virion/Serion) for $2 \mathrm{~h}$ at $37^{\circ} \mathrm{C}$ in a humid chamber. After washing, antigen-antibody complexes were incubated in the presence of $1.0 \mathrm{M}$ ammonium thiocyanate or PBS as control for $10 \mathrm{~min}$ at $37^{\circ} \mathrm{C}$. After washing to remove antibodies bound with lowavidity, the ELISA was completed according to the manufacturer's instructions. The relative avidity index was calculated as follows: IgG concentrations (NH4SCN) / IgG concentrations 270 (PBS) $\times 100$ and is given in percent ${ }^{21,22}$.

\section{SARS-CoV-2 neutralization assay}

High-titer virus stocks were generated by infection of Vero-E6 cells (American Type Culture Collection, ATCC, USA) grown in virus expansion medium (Dulbecco's Modified Eagle's Medium containing $5 \%$ fetal bovine serum, $100 \mathrm{U} / \mathrm{mL}$ penicillin-streptomycin). Cells were incubated with clinical isolates of different SARS-CoV-2 variants (GISAID EPI ISL: 2450298 [EU1/B.1.177], 2095258 [alpha/B.1.1.7], 1752394 [beta/B.1.351], 2095178 [gamma/P.1/B.1.1.28.1], 2772700 [delta/B.1.617.2], 7808190 [omicron/B.1.1.529]). EU1 and the omicron $\mathrm{VoC}$ were isolated from nasopharyngeal swabs of COVID-19 patients. Isolates of VoCs alpha, beta, gamma and delta were kindly provided by the Bavarian Landesamt für Gesundheit und Lebensmittel. Virus stocks were expanded by two passages before harvest and stored at $-80^{\circ} \mathrm{C}$. All virus stocks were only used for infection experiments after sequencing of the complete viral genomes. Virus stocks were characterized by rRT-PCR as reported previously ${ }^{23}$.

For each individual SARS-CoV-2 VoC, the tissue culture infectious dose resulting in $90 \%$ loss of target cell viability $48 \mathrm{~h}$ after infection $\left(\mathrm{TCID}_{90}\right)$ was determined using a dilution series of the virus stock on MDA-MB-231 cells (ATCC) overexpressing the human angiotensin-converting enzyme 2 receptor (MDA-MB-231-hACE2). For infection neutralization, cells were cultured and infected in 384-well plates (7,500 cells/well). The respective $\mathrm{TCID}_{90}$ of each virus stock, was incubated for $2 \mathrm{~h}$ with different concentrations of each serum to be tested. Subsequently, $10 \mu \mathrm{L}$ of the virus-serum mixtures were added to $20 \mu \mathrm{L}$ medium and added to MDA-MB-231-hACE2 
cells. $48 \mathrm{~h}$ post infection, cytopathic effects were recorded by addition of $10 \mu \mathrm{L}$ CellTiter-Glo 2.0 reagent (Promega, Wisconsin, USA) and subsequent measurement of bioluminescence signals ( $0.5 \mathrm{~s}$ integration time, no filter) to quantify virus-mediated killing of target cells.

\section{Data analysis}

Data and statistical analyses were performed in Prism 9 (GraphPad Software, California, USA). TCID 90 values for tissue culture infectious doses and IC $\mathrm{I}_{50}$ values for neutralization were calculated after normalized, sigmoidal dose response curve approximation of the respective data.

\section{Data availability}

All data obtained in this study are available in the source data accompanying the manuscript.

\section{Acknowledgements}

We thank the BioSysM liquid handling unit for support. We thank the entire team of the COVID19 vaccination center of the University Hospital München rechts der Isar (MRI) and the study team as well as Timo Vogt for their help and appreciate the efforts of Alexander Herrmann and Romina Bester to maintain the BSL3 lab.

\section{Author contributions}

Conceptualization: P.A.K., O.T.K., U.P.

Methodology: P.R.W., M.S., A.P., A.W., E.V., N.G., H.M., M.F., C-C.C., G.L., M.A., E.M-P., M.M., S.K., H.M., C.K., J.W., D.H., S.K., G.A., T.V., M.P., O.T.K.

Resources: S.B., P.S., V.F.

315 Formal Analysis: C.D., M.M., A.G., S.J., N.G., H.M., M.W., E.V., S.Y., K.T., C.K.

Writing - Original Draft: P.R.W., A.P., P.A.K., O.T.K., U.P.

Writing - Review \& Editing: all authors.

Visualization: P.R.W., A.P, P.K., O.T.K., U.P. 
Supervision: H.B., V.H., B.L., K.U., P.K., O.T.K., U.P.

Funding Acquisition: B.L., K.U., P.K., O.T.K., U.P.

\section{Funding}

This study was supported by: BMBF initiative NaFoUniMedCovid19 (01KX2021), subproject B-FAST (P.A.K., O.T.K., U.P.), the Bavaria-Saxony research alliance FOR-COVID (M.M., O.T.K., U.P.), the Bavarian research network Bay-VOC (M.M., O.T.K., K.Ü., U.P.), the CoViPaconsortium of the Helmholtz association (U.P. and P.A.K), the CoVRapid consortium (U.P. and P.A.K.) and the CoVaKo study (to K.Ü. and U.P.).

\section{Declaration of interests}

330 The authors declare to have no conflict of interest concerning the study content. Outside of the study they declare the following interests: UP is co-founder, share-holder and board member of SCG Cell therapy, member of the scientific advisory board of Leukocare and member of topic-specific scientific advisory boards of Sanofi-Pasteur, GILEAD and GSK and ad hoc advisor for BioNTech (without remuneration).

\section{References}

1. Mohapatra, R.K., et al. Omicron (B.1.1.529 variant of SARS-CoV-2); an emerging threat: current global scenario. J Med Virol (2021).

2. Volz, E., et al. Assessing transmissibility of SARS-CoV-2 lineage B.1.1.7 in England. Nature 593, 266269 (2021).

3. Tegally, H., et al. Detection of a SARS-CoV-2 variant of concern in South Africa. Nature 592, 438-443 (2021).

4. Fujino, T., et al. Novel SARS-CoV-2 Variant in Travelers from Brazil to Japan. Emerg Infect Dis 27(2021).

5. Mlcochova, P., et al. SARS-CoV-2 B.1.617.2 Delta variant replication and immune evasion. Nature 599, 114-119 (2021).

6. Wang, Z., et al. mRNA vaccine-elicited antibodies to SARS-CoV-2 and circulating variants. Nature 592, 616-622 (2021).

7. Planas, D., et al. Reduced sensitivity of SARS-CoV-2 variant Delta to antibody neutralization. Nature 596, 276-280 (2021).

8. Karim, S.S.A. \& Karim, Q.A. Omicron SARS-CoV-2 variant: a new chapter in the COVID-19 pandemic. 
Lancet 398, 2126-2128 (2021).

9. Schmidt, F., et al. High genetic barrier to SARS-CoV-2 polyclonal neutralizing antibody escape. Nature 600, 512-516 (2021).

10. Khoury, D.S., et al. Neutralizing antibody levels are highly predictive of immune protection from symptomatic SARS-CoV-2 infection. Nat Med 27, 1205-1211 (2021).

11. Muecksch, F., et al. Affinity maturation of SARS-CoV-2 neutralizing antibodies confers potency, breadth, and resilience to viral escape mutations. Immunity 54, 1853-1868 e1857 (2021).

12. Victora, G.D. \& Nussenzweig, M.C. Germinal Centers. Annual Review of Immunology 30, 429-457 (2012).

13. Chia, W.N., et al. Dynamics of SARS-CoV-2 neutralising antibody responses and duration of immunity: a longitudinal study. The Lancet Microbe 2, e240-e249 (2021).

14. Weinberger, T., et al. Prospective Longitudinal Serosurvey of Healthcare Workers in the First Wave of the Severe Acute Respiratory Syndrome Coronavirus 2 (SARS-CoV-2) Pandemic in a Quaternary Care Hospital in Munich, Germany. Clin Infect Dis 73, e3055-e3065 (2021).

15. Muenchhoff, M., et al. Genomic epidemiology reveals multiple introductions of SARS-CoV-2 followed by community and nosocomial spread, Germany, February to May 2020. Euro Surveill 26(2021).

16. Gaebler, C., et al. Evolution of antibody immunity to SARS-CoV-2. Nature 591, 639-644 (2021).

17. Sokal, A., et al. Maturation and persistence of the anti-SARS-CoV-2 memory B cell response. Cell 184, 1201-1213 e1214 (2021).

18. Collie, S., Champion, J., Moultrie, H., Bekker, L.G. \& Gray, G. Effectiveness of BNT162b2 Vaccine against Omicron Variant in South Africa. N Engl J Med (2021).

19. Nemet, I., et al. Third BNT162b2 Vaccination Neutralization of SARS-CoV-2 Omicron Infection. N Engl $J$ Med (2021).

20. Saadat, S., et al. Binding and Neutralization Antibody Titers After a Single Vaccine Dose in Health Care Workers Previously Infected With SARS-CoV-2. JAMA 325, 1467-1469 (2021).

21. Almanzar, G., Ottensmeier, B., Liese, J. \& Prelog, M. Assessment of IgG avidity against pertussis toxin and filamentous hemagglutinin via an adapted enzyme-linked immunosorbent assay (ELISA) using ammonium thiocyanate. J Immunol Methods 387, 36-42 (2013).

22. Kneitz, R.H., et al. A new method for determination of varicella-zoster virus immunoglobulin $G$ avidity in serum and cerebrospinal fluid. BMC Infect Dis 4, 33 (2004).

23. Muenchhoff, M., et al. Multicentre comparison of quantitative PCR-based assays to detect SARS-CoV2, Germany, March 2020. Euro Surveill 25(2020). 
- Wratil et al: Superior immunity allows neutralization of all SARS-CoV-2 VoCs -

\section{Figure 1}
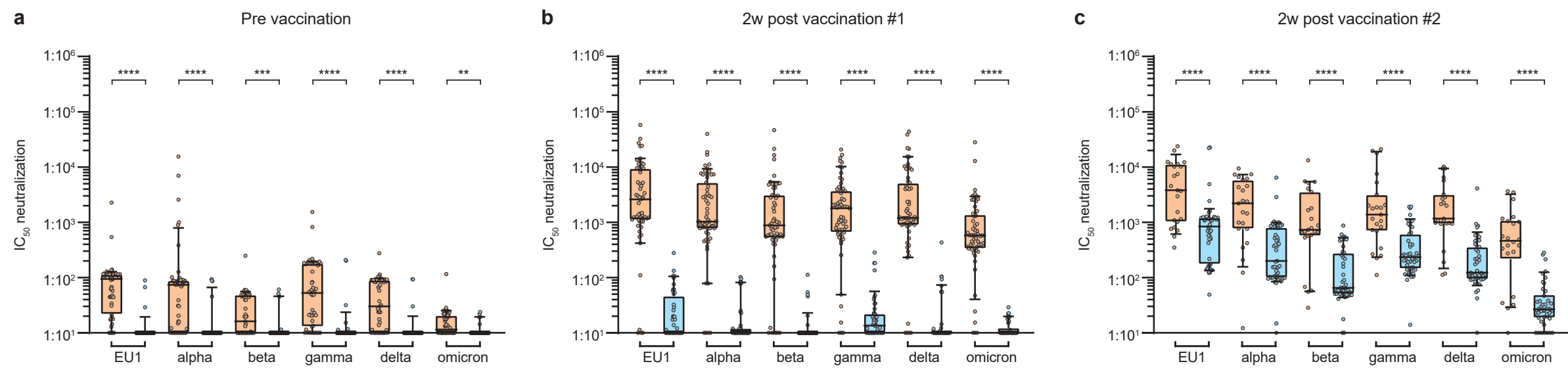

d

$7 \mathrm{~m}$ post vaccination \#2

e

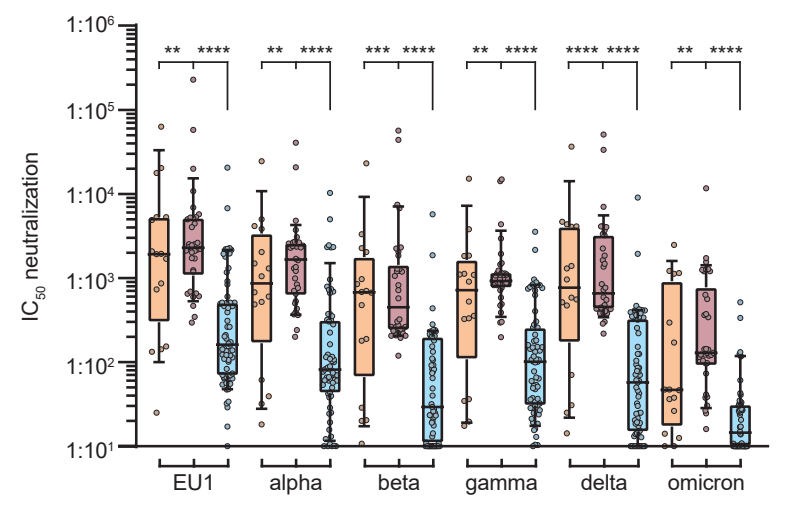

$7 \mathrm{~m}$ post vaccination \#2

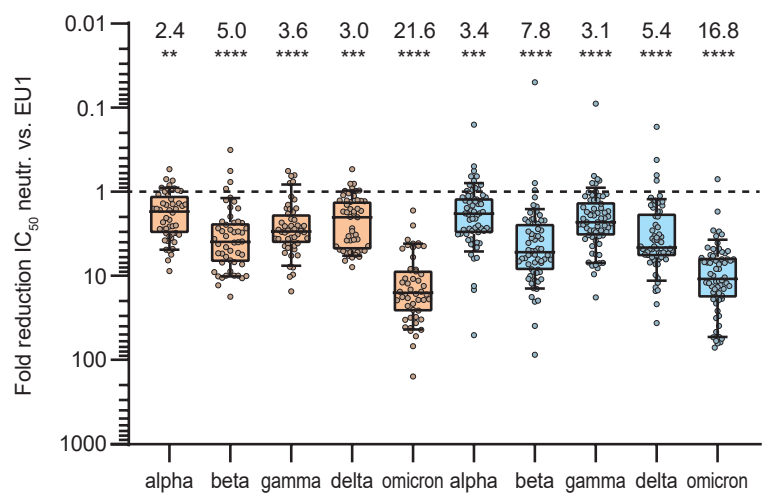

2w post vaccination \#3

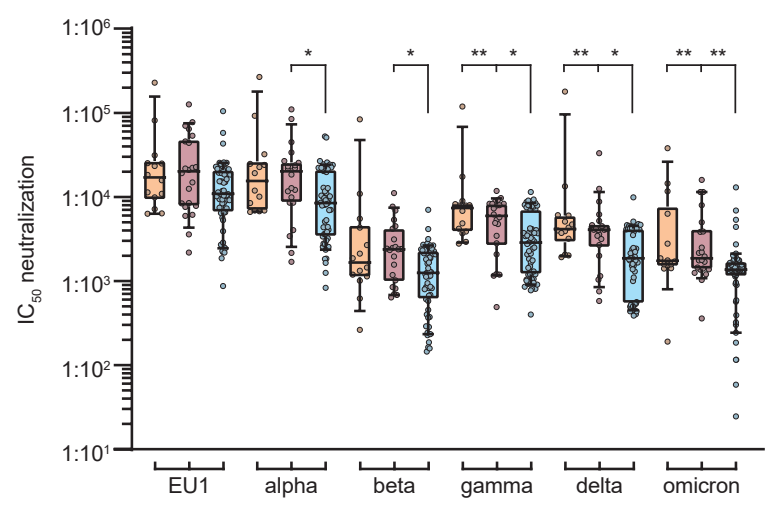

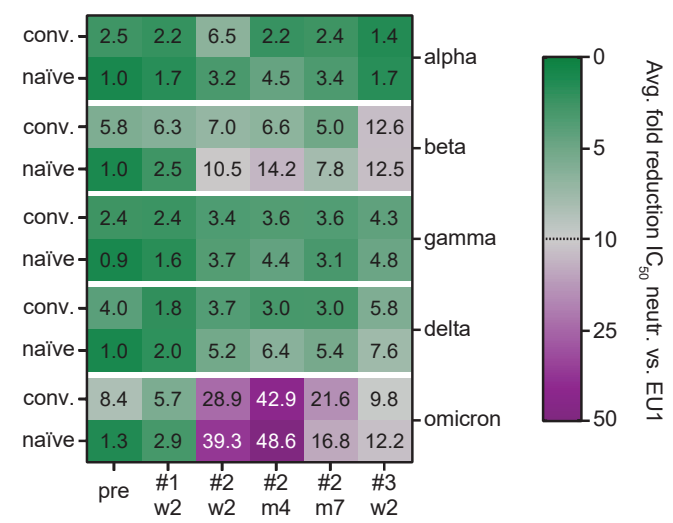

$\square$ convalescent $\square$ convalescents w/o vacc. \#2 $\quad \square$ naïve 
Figure 1. Comparison of infection neutralization activities for SARS-CoV-2 variants in naïve individuals and SARS-CoV-2 convalescents at different time points following mRNA BNT162b2 vaccination

SARS-CoV-2 convalescents (orange), and naïve participants (blue) are shown at indicated time points before and after vaccination. (d,f) SARS-CoV-2 convalescents who received only vaccination \#1 and \#3 are separated (red). (a-d, f) Half maximal inhibitory concentrations $\left(\mathrm{IC}_{50}\right)$ for serum infection-neutralization of SARS-CoV-2 strain EU1 and VoC alpha, beta, gamma, delta and omicron normalized to $10^{7}$ viral RNA copies are shown as box plots with whiskers between the $10^{\text {th }}$ and $90^{\text {th }}$ percentile. Values in brackets indicate samples tested against omicron. (a) sera from 51 (50) SARS-CoV-2 convalescents collected approx. 9 months after infection and 34 (29) SARS-CoV-2 naïve individuals prior to vaccination (pre); (b) sera from 59 (56) convalescents and 48 (42) naïves collected 2 weeks after vaccination \#1 (w2); (c) sera from 23 (22) convalescents and 47 (42) naïves collected two weeks after vaccination \#2; (d) sera from 16 convalescents and 65 (64) naïves collected 7 months ( $\mathrm{m} 7$ ) after vaccination \#2 and 34 convalescents having received only vaccination \#1 collected in parallel; (e) fold-reductions of $\mathrm{IC}_{50}$ values comparing neutralization of EU1 with that of indicated SARS-CoV-2 VOCs depicted as box plots with whiskers between the $10^{\text {th }}$ and $90^{\text {th }}$ percentile in 50 convalescents and 64 naïves (blue) 7 months after vaccination \#2. Numbers above boxes indicate average fold changes comparing EU1 and SARS-CoV-2 VOCs. (f) sera from 14 convalescents and 59 naïve participants collected 2 weeks after vaccination \#3 as well as 22 convalescents who received only vaccination \#1 and \#3; (g) heatmap indicating the average (Avg.) fold-reduction of $\mathrm{IC}_{50}$ values for the indicated $\mathrm{VoC}$ compared to $\mathrm{IC}_{50}$ values for EU1 in convalescent (conv.) and naïve participants at the different time points. Statistics were done using Mann-Whitney test (a-c), Kruskal-Wallis-test with Dunn's multiple testing correction (d, f) and Friedman test with Dunn's multiple testing correction (e). Connecting lines indicate statistically significant differences between groups. ${ }^{*} p \leq 0.05,{ }^{* *} p \leq 0.01,{ }^{* * *} p \leq 0.001,{ }^{* * *} p \leq 0.0001$. If no connecting lines or asterisks are given, no significant differences were detected. m4: 4 months after vaccination \#2. 


\section{Figure 2}

a

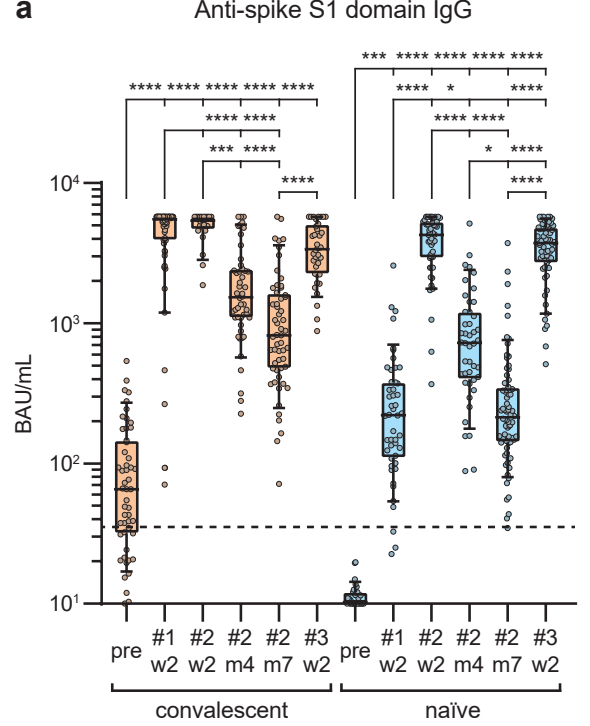

Omicron

Avg. fold difference $\mathrm{IC}_{50}$ neutralization

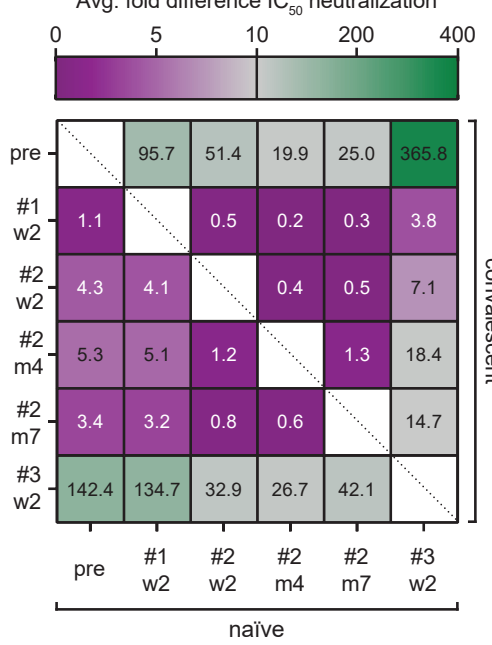

b

Delta

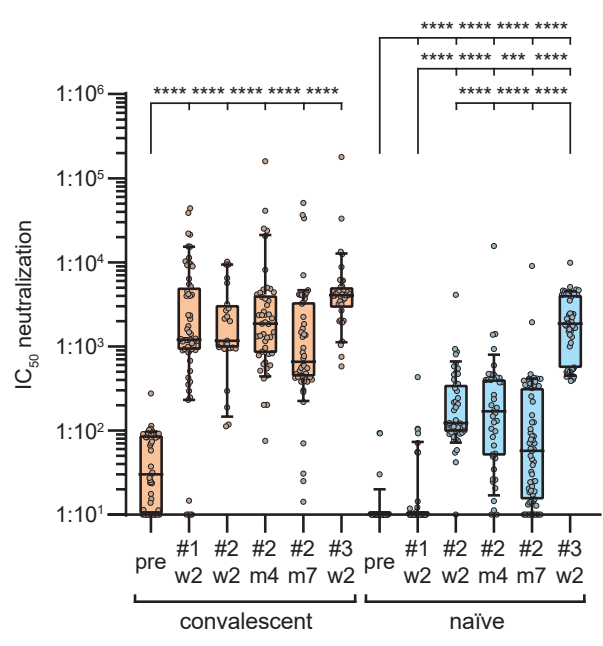

Delta

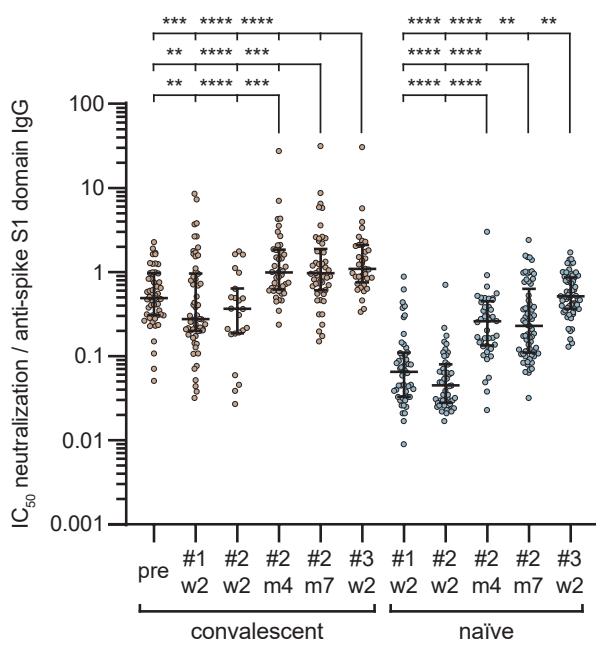

Omicron

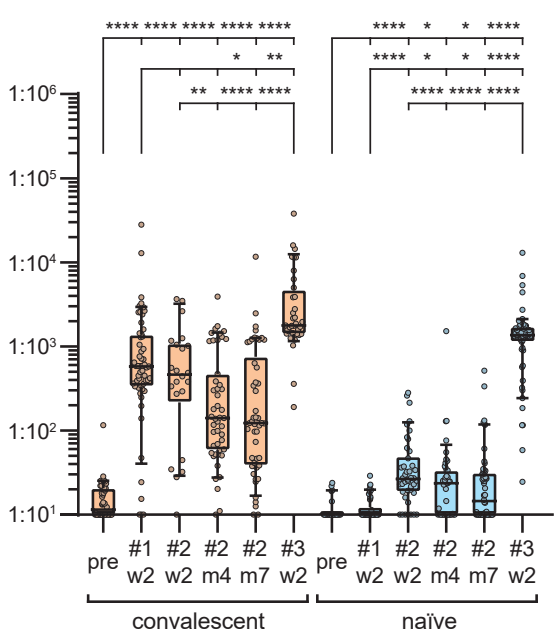

g

Omicron

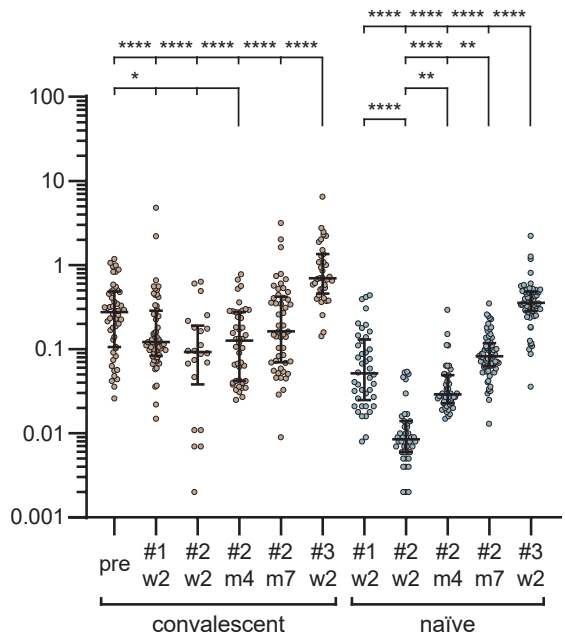

d

Delta

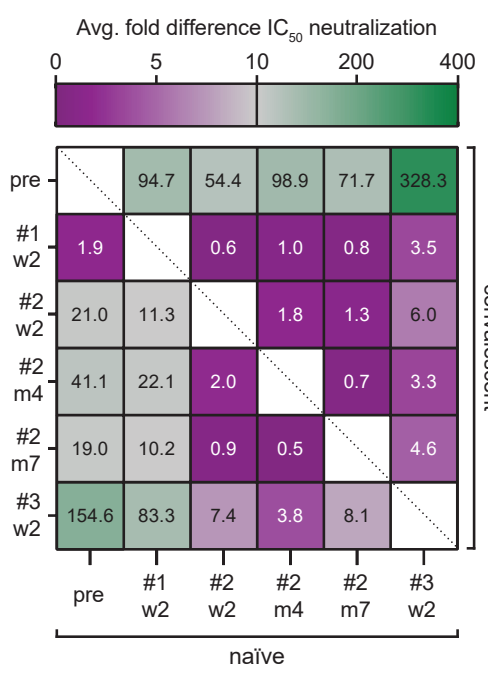

IgG avidity

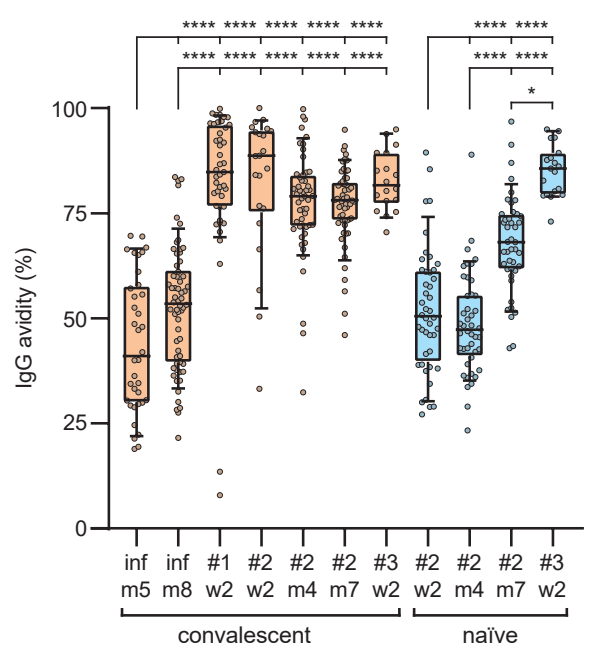

$\square$ convalescent

$\square$ naïve 
Figure 2. Longitudinal analysis of antibody titers, infection neutralization of delta and omicron VoCs and antibody avidity in naïve individuals and convalescents following mRNA BNT162b2 vaccination

(a) anti-spike $\$ 1$ domain lgG titres in 274 sera from 62 convalescents, and 304 sera from 73 naïve participants are given as binding arbitrary units $(B A U) / m L$. (b, c) half maximal inhibitory concentrations $\left(\mathrm{IC}_{50}\right)$ for serum infection neutralization normalized to $10^{7}$ viral RNA copies of SARS-CoV-2 VoCs delta in $266 / 296$ (b) and omicron and $261 / 279$ (c) sera from 62 convalescents / 73 naïves, respectively. (d, e) heatmaps showing average fold-changes in $\mathrm{IC}_{50}$ values for delta (d) and omicron (e) between the respective time points for convalescent and naïve individuals. ( $\mathbf{f}, \mathbf{g}$ ) ratios between infection neutralization $\mathrm{IC}_{50}$ values and anti-spike $\mathrm{S} 1$ domain antibody titers for delta in 263 / 295 (f) and for omicron in 258 / 278 sera from 62 convalescents / 73 naïves, respectively. (h) IgG-type anti-spike antibody avidities in 288 sera from 90 convalescents, and 150 sera from 47 naïves. (a,b,c,h) box plots with whiskers between the $10^{\text {th }}$ and $90^{\text {th }}$ percentile are given, SARS-CoV-2 convalescents (orange) and naïve participants (blue). $(\mathbf{d}, \mathbf{e})$ medians and interquartile ranges are shown. Differences between time points were analyzed for their statistical significance using the Kruskal-Wallis test with Dunn's multiple testing correction. Connecting lines indicate statistically significant differences between groups: ${ }^{*} p \leq 0.05$, ${ }^{* *} p \leq 0.01,{ }^{* * *} p \leq 0.001,{ }^{* * * *} p \leq 0.0001$. If no connecting lines or asterisks are given, no significant differences were detected.

pre: prior to first vaccination; \#1 - first vaccination; \#2 - second vaccination; \#3 - third vaccination; w2 - two weeks after respective vaccination; $\mathrm{m} 4-4$ months after vaccination; $\mathrm{m} 7-7$ months after vaccination. 


\section{Figure 3}

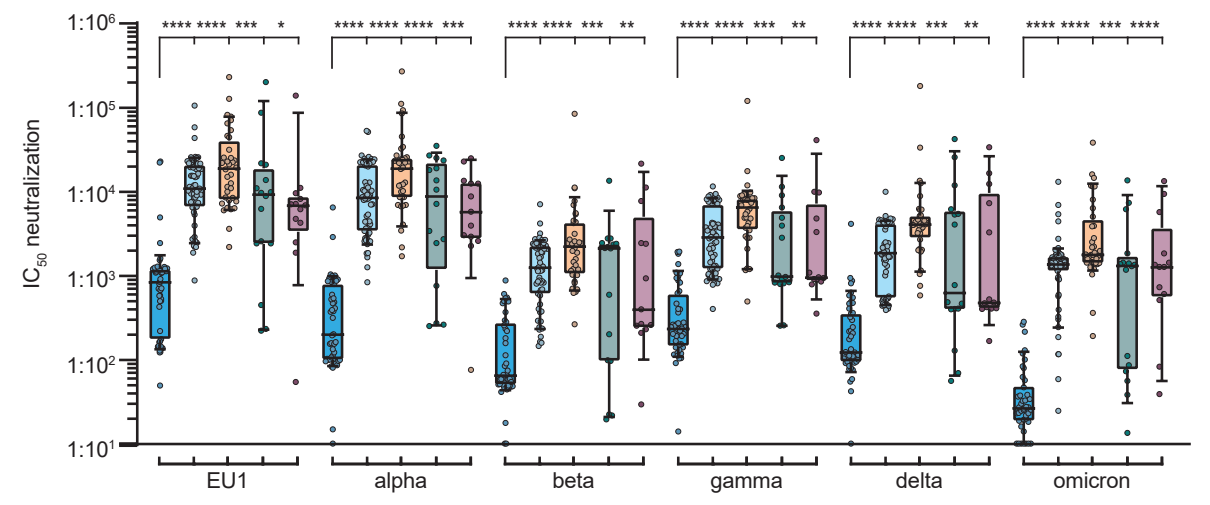

$\square$ naïves 2w post vacc. \#2

$\square$ naïves $2 \mathrm{w}$ post vacc. \#3

$\square$ convalescents 2 w post vacc. \#3

$\square$ delta breakthrough

$\square$ omicron breakthrough 
Figure 3. Infection neutralization capacity for SARS-CoV-2 variants in COVID-19 patients after breakthrough infection compared to vaccinated naïve individuals and SARS-CoV-2 convalescents.

Half maximal inhibitory concentrations $\left(\mathrm{IC}_{50}\right)$ for serum infection-neutralization normalized to $10^{7}$ viral RNA copies of SARS-CoV-2 variants are shown as box plots with whiskers between the $10^{\text {th }}$ and $90^{\text {th }}$ percentile in 47 naïve participants (42 in case of omicron) 2 weeks after vaccination \#2 (dark blue), 59 naïve (light blue) and 36 convalescent participants 2 weeks after vaccination \#3, as well as 16 and 13 vaccinated individuals on average 7 days after PCR-confirmed breakthrough infections with delta (green) or omicron (purple), respectively. Differences between groups were analyzed for their statistical significance using the Kruskal-Wallis test with Dunn's multiple testing correction. Connecting lines indicate statistically significant differences between groups: ${ }^{\star} p \leq 0.05,{ }^{* *} p \leq 0.01,{ }^{* \star *} p \leq 0.001,{ }^{* * * *} p \leq 0.0001$. If no connecting lines or asterisks are given, no significant differences were detected. 
Extended Data Table 1. Longitudinal cohort characteristics of naïve and convalescent participants (cohort 1 )

\begin{tabular}{ll}
\hline $\begin{array}{l}\text { Naïve individuals } \\
\text { cohort (1) }\end{array}$ & \\
\hline \hline participants - $n$ & 73 \\
$\quad$ one initial vaccination & 0 \\
$\quad$ two initial vaccinations & 73 \\
vaccine received & BNT162b2 \\
time span - median days (IQR) & \\
$\quad$ vaccination \#1 to \#2 & 22 \\
vaccination \#2 to \#3 & $272(265$ to 282$)$ \\
$\quad$ female & \\
male & $65.8 \%$ \\
age - median years (IQR) & $34.2 \%$ \\
\end{tabular}

\begin{tabular}{ll}
\hline $\begin{array}{l}\text { Convalescent individuals } \\
\text { (cohort 1) }\end{array}$ & \\
\hline \hline & 98 \\
participants - $n$ & 68 \\
thereof vaccinated & 43 \\
$\quad$ one initial vaccination & 25 \\
two initial vaccinations & 6 \\
thereof excluded due to potential SARS-CoV-2 re-exposure & March 2020 to April 2020 \\
time period of SARS-CoV-2 infection & 0 \\
hospitalizations - $n$ & BNT162b2 \\
vaccine received & 21 \\
\hline $\begin{array}{l}\text { time span - median days (IQR) } \\
\text { vaccination \#1 to \#2 } \\
\text { vaccination \#1/\#2 to \#3 }\end{array}$ & $259(237$ to 268$)$ \\
gender - $\%$ & \\
female & $54.1 \%$ \\
male & $45.9 \%$ \\
age - median years (IQR) & 38 (29 to 53$)$ \\
\hline
\end{tabular}


Extended Data Table 2. Cohort characteristics of vaccinated individuals with breakthrough infection (cohort 2)

Individuals with breakthrough infection

(cohort 2)

participants - $n$

delta breakthrough

omicron breakthrough

time period of SARS-CoV-2 infection

hospitalisations - $n$

vaccines received

$2 \times$ BNT162b2 - $n$

delta breakthrough

omicron breakthrough

$2 \times$ mRNA-1273 - $n$

delta breakthrough

omicron breakthrough

2 x ChAdOx 1 - $n$

delta breakthrough

omicron breakthrough

$1 \times$ ChAdOx1 + 1 x BNT162b2 - $n$

delta breakthrough

omicron breakthrough

$1 \times$ Ad26.COV2.S - $n$

delta breakthrough

omicron breakthrough

time span - median days (IQR)

delta breakthrough

initial vaccinations to first positive PCR result

first positive PCR result to sample collection

omicron breakthrough

initial vaccinations to first positive $\mathrm{PCR}$ result

first positive PCR result to sample collection

gender - \%

delta breakthrough

female

male

omicron breakthrough

female

male

age - median years (IQR)

delta breakthrough

omicron breakthrough
29

16

13

July 2021 to December 2021

0

20

12

8

4

2

2

2

0

2

2

2

0

1

0

1

141 (99 to 242)

7 (5 to 7 )

172 (156 to 207)

10 (7 to 10$)$

$56.3 \%$

$43.7 \%$

$38.5 \%$

$61.5 \%$

35 (31 to 38 )

42 (28 to 52 ) 

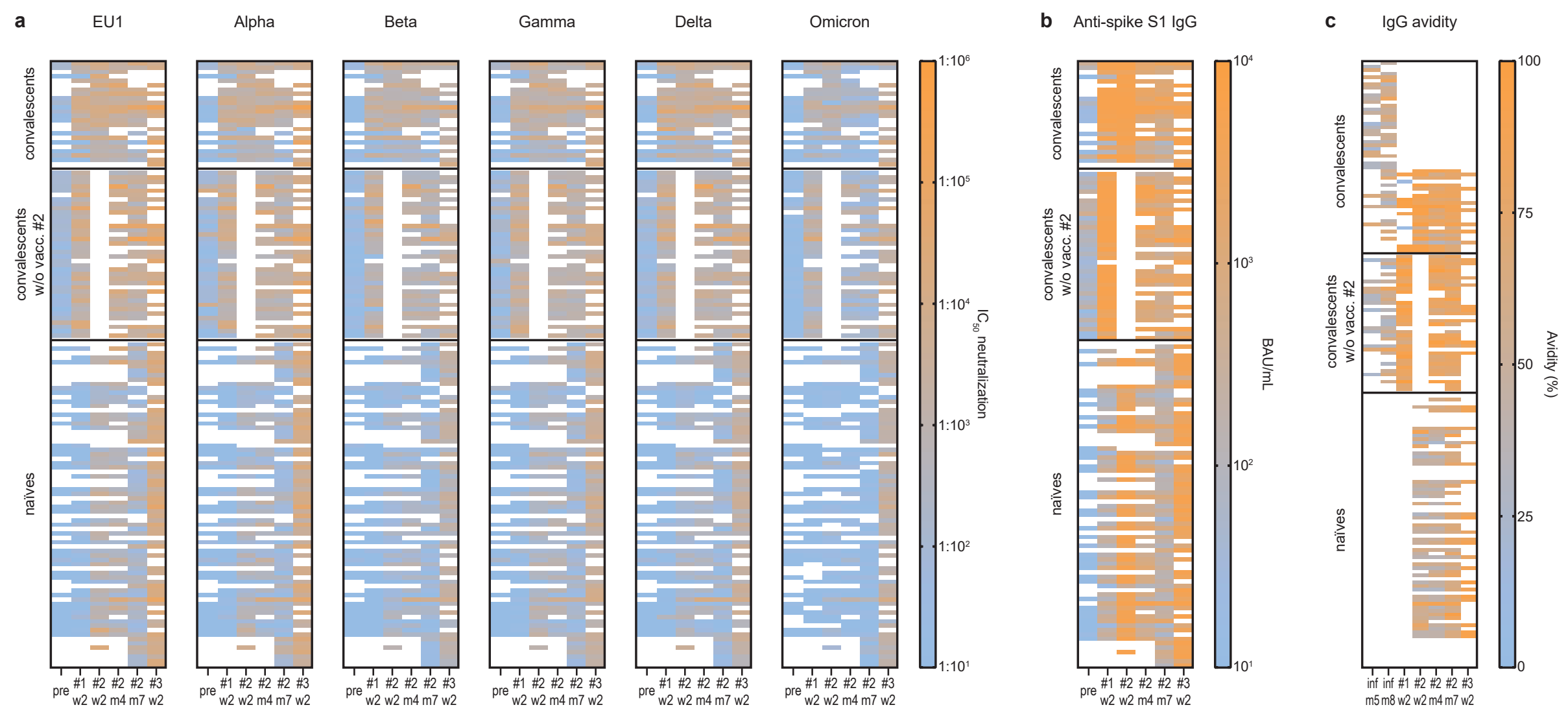


\section{Extended Data Figure 1. Heatmaps of infection neutralization activities against SARS-CoV- 2 variants, anti-SARS-CoV-2 antibody responses, and antibody avidity in naïve individuals and convalescents following BNT162b2 vaccination}

$(\mathbf{a}, \mathbf{b})$ heatmaps of half maximal inhibitory concentrations $\left(\mathrm{IC}_{50}\right)$ normalized to $10^{7}$ viral RNA copies for serum infection-neutralization of SARS-CoV-2 variants (a) and IgG-type anti-spike S1 domain antibody titers (b) in 24 SARS-CoV-2 convalescents, 38 convalescents who did not receive vaccination \#2, and 73 naïve participants. (c) heatmap of IgG-type anti-spike antibody avidity in 54 SARS-CoV-2 convalescents, 38 convalescents who did not receive the second vaccination, and 73 naïve participants. For white areas within heatmaps data was not available. pre - prior to first vaccination; \#1 - first vaccination time point; \#2 - second vaccination; \#3 - third vaccination; w2 - two weeks after respective vaccination; $\mathrm{m} 4-4$ months after vaccination; $\mathrm{m} 7-7$ months after vaccination; inf. $\mathrm{m} 5-5$ months after SARS-CoV-2 infection; inf. m8 - 8 months after SARSCoV-2 infection; BAU - binding antibody units. 
Extended Data Figure 2

4m post vaccination \#2

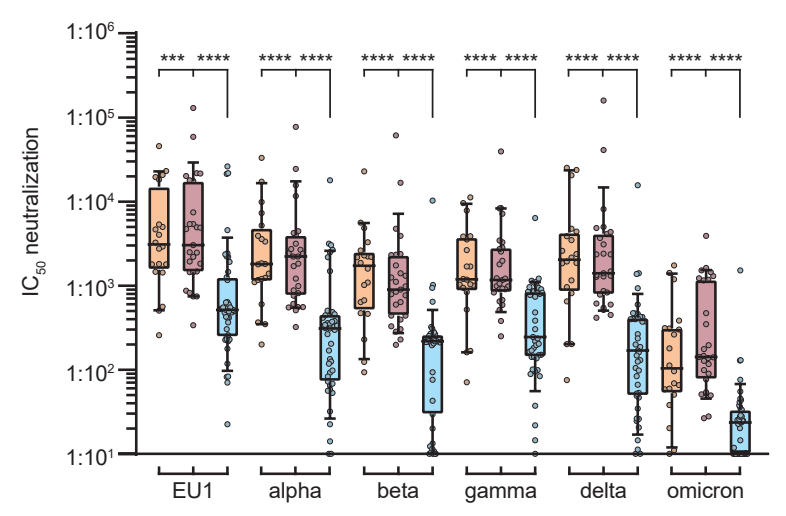

$\square$ convalescents $\square$ convalescents w/o vacc. \#2

$\square$ naïves 


\section{Extended Data Figure 2. Comparison of infection neutralization activities for SARS-CoV-2 variants in naïve individuals and SARS-CoV-2 convalescents 4 months after BNT162b2 vaccination}

Half maximal inhibitory concentrations $\left(\mathrm{IC}_{50}\right)$ normalized to $10^{7}$ viral RNA copies for serum infection-neutralization of SARS-CoV-2 variants EU1, alpha, beta, gamma, delta and omicron are shown as box plots with whiskers between the $10^{\text {th }}$ and $90^{\text {th }}$ percentile for 20 SARS-CoV-2 convalescents (orange), and 43 naïve participants (blue) collected 4 months after the second vaccination as well as 27 convalescents who did not receive vaccination \#2 collected at the same time point (red). Differences in the $\mathrm{IC}_{50}$ values were analyzed for statistical significance using the Kruskal-Wallis-test with Dunn's multiple testing correction. Connecting lines indicate statistically significant differences between groups: ${ }^{*} p \leq 0.05,{ }^{* *} p \leq 0.01,{ }^{* * * *} p \leq 0.001,{ }^{* * * * *} p \leq 0.0001$. If no connecting lines or asterisks are given, no significant differences were detected. 
Wratil et al: Superior immunity allows neutralization of all SARS-CoV-2 VoCs -

\section{Extended Data Figure 3}

a

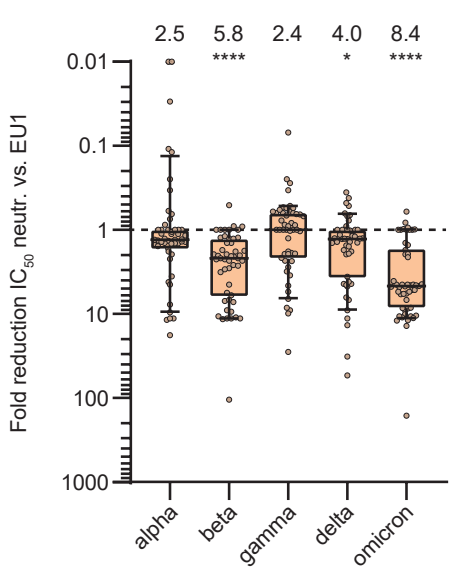

d

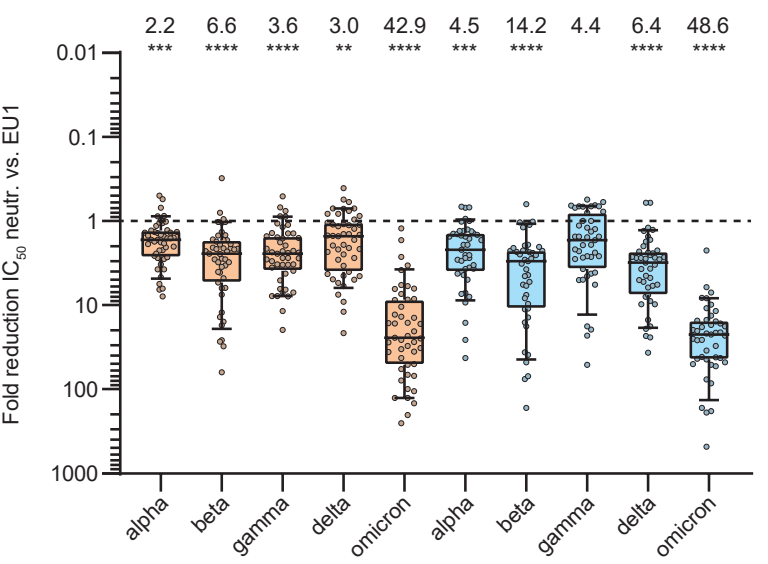

b

2w post vaccination \#1

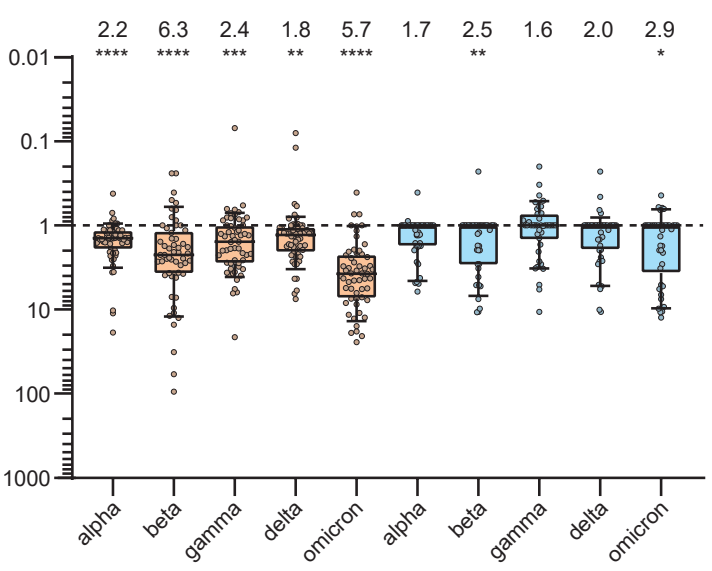

e

$7 \mathrm{~m}$ post vaccination \#2

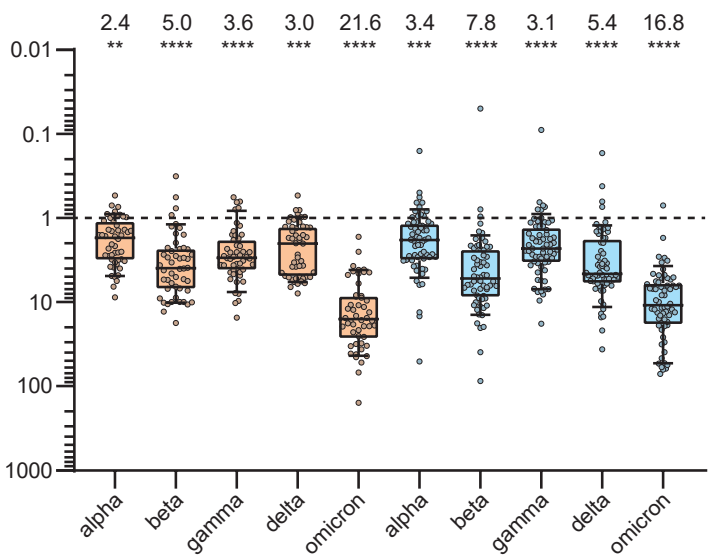

2w post vaccination \#2

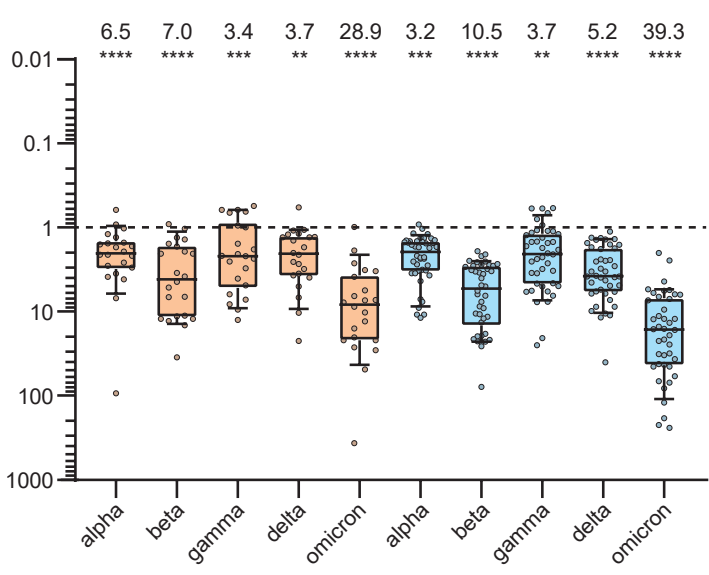

f

2w post vaccination \#3

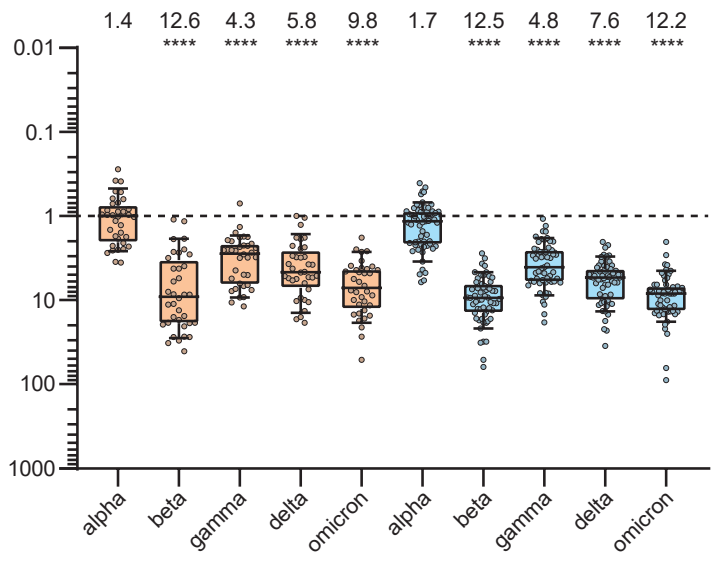




\section{Extended Data Figure 3. Comparison of infection-neutralization activities for SARS-CoV-2 variants in naïve individuals and SARS-CoV-2 convalescents at different time points following BNT162b2 vaccination}

The fold reductions of the half maximal inhibitory concentrations $\left(\mathrm{IC}_{50}\right)$ comparing the neutralization of EU1 with the other SARS-CoV-2 VOCs are depicted in as box plots with whiskers between the $10^{\text {th }}$ and $90^{\text {th }}$ percentile SARS-CoV-2 convalescents (orange) and 64 naïve participants (blue). (a) sera from 50 SARS-CoV-2 convalescents collected prior to vaccination; (b) sera from 56 convalescents, and 42 naïves collected 2 weeks after the first vaccination; (c) sera from 22 convalescents, and 42 naïves collected 2 weeks after the second vaccination; (d) sera from 47 convalescents, and 43 naïves collected 4 months after the second vaccination; (e) sera from 50 convalescents, and 64 naïves collected 7 months after the second vaccination; (f) sera from 36 convalescents, and 59 naïves collected 2 weeks after the third vaccination. Differences in fold changes were analyzed for their statistical significance using the Friedman test with Dunn's multiple testing correction Connecting lines indicate statistically significant differences between groups: ${ }^{*} p \leq 0.05,{ }^{* *} p \leq 0.01,{ }^{* * *} p \leq 0.001,{ }^{* * * * *} p \leq 0.0001$. If no connecting lines or asterisks are given, no significant differences were detected. Numbers above boxes indicate average fold changes comparing EU1 and the respective SARS-CoV-2 VOC. 


\section{Extended Data Figure 4}

a

Anti-spike S1 domain lgG

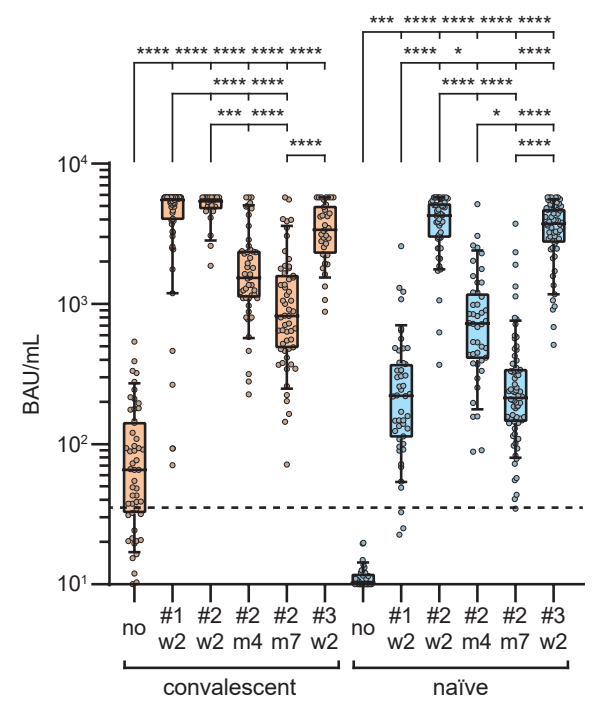

e
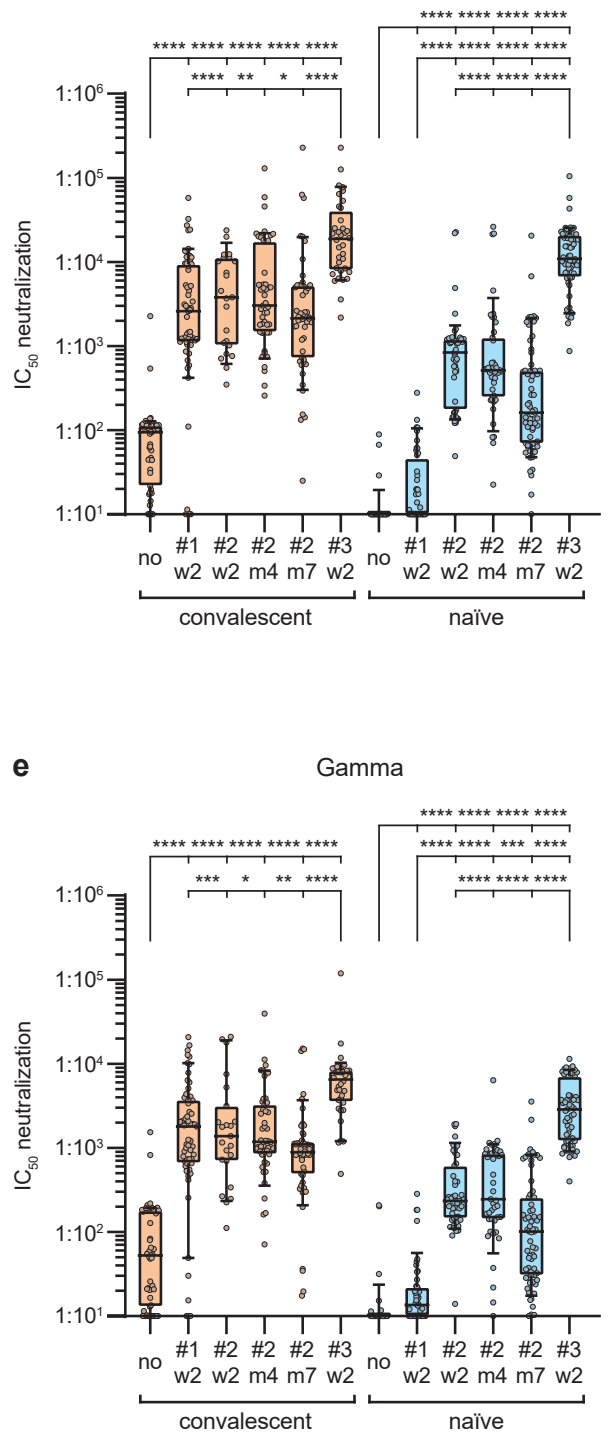

c

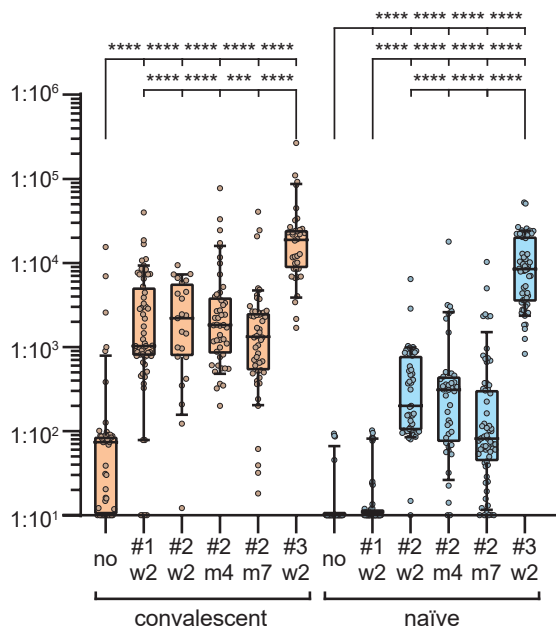

f

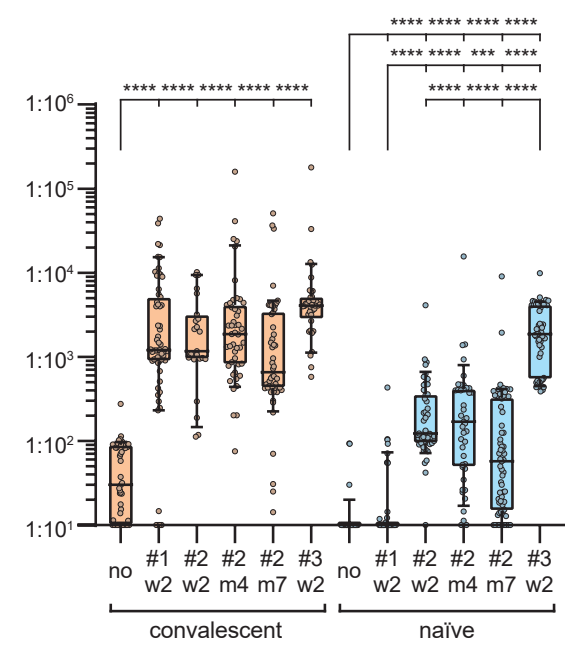

Beta
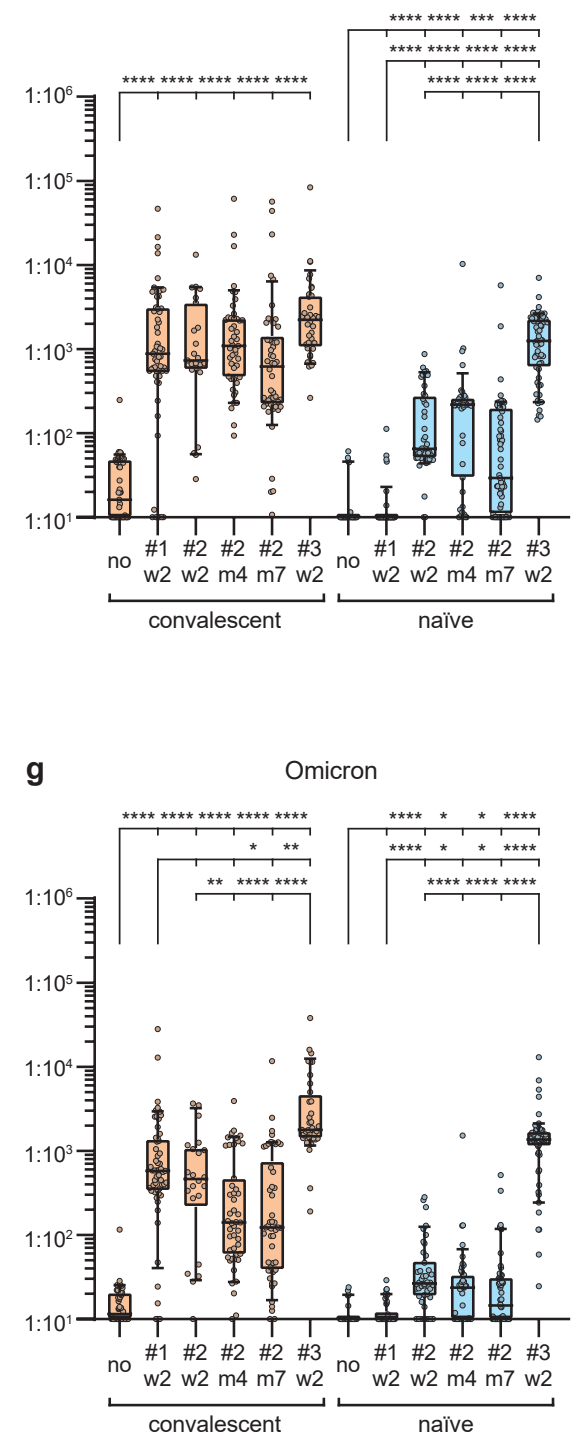


\section{Extended Data Figure 4. Longitudinal comparison of infection neutralization activities against SARS-CoV-2 variants and anti-SARS-CoV-2 antibody responses in naïve individuals and convalescents following BNT162b2 vaccination}

(a) anti-spike S1 domain antibody titers in 274 sera from 62 convalescents, and 304 sera from 73 naïve participants were analyzed are given as binding arbitrary units (BAU)/mL. (b-g) Half maximal inhibitory concentrations $\left(\mathrm{IC}_{50}\right)$ for serum infection neutralization normalized to $10^{7}$ viral RNA copies of SARS-CoV-2 VoCs EU1, alpha, beta, gamma, and delta in 266 / 296 (b-f) and omicron and 261 / 279 (g) sera from 62 convalescents / 73 naïves, respectively. Data are shown as box plots with whiskers between the $10^{\text {th }}$ and $90^{\text {th }}$ percentile for SARS-CoV-2 convalescents (orange) and naïve participants (blue). Differences between time points were analyzed for their statistical significance using the Kruskal-Wallis test with Dunn's multiple testing correction. Connecting lines indicate statistically significant differences between groups: ${ }^{*} p \leq 0.05,{ }^{* *} p \leq 0.01,{ }^{* * *} p \leq 0.001$, ${ }^{* * * * *} p \leq 0.0001$. If no connecting lines or asterisks are given, no significant differences were detected.

pre: prior to first vaccination; \#1 - first vaccination; \#2 - second vaccination; \#3 - third vaccination; w2 - two weeks after respective vaccination; $\mathrm{m} 4-4$ months after vaccination; $\mathrm{m} 7$ - 7 months after vaccination. 
a
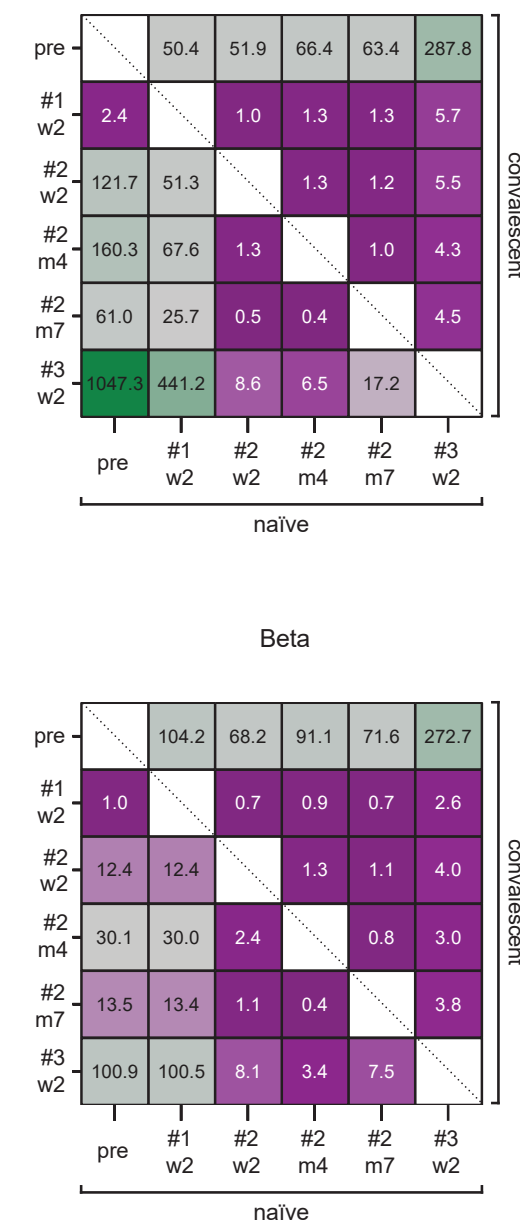

b

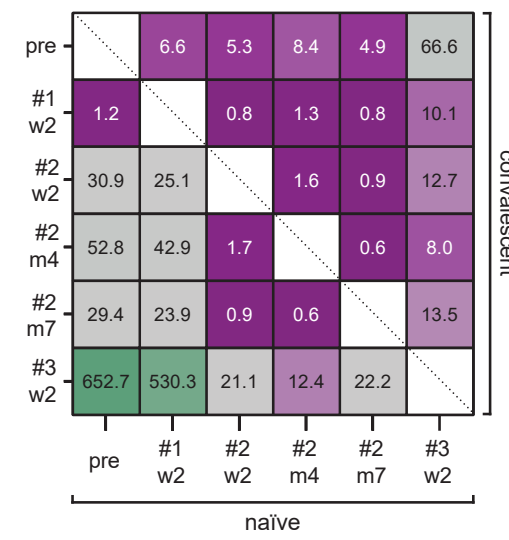

d

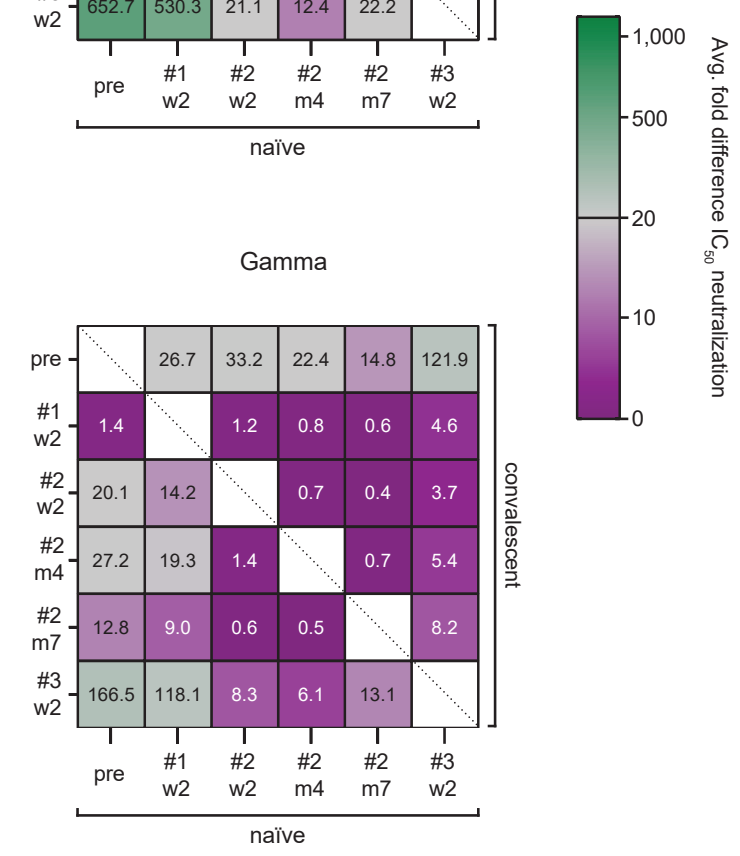




\section{Extended Data Figure 5. VoC-centered heatmaps for longitudinal comparisons of infection neutralization activities in naïve individuals and convalescents following BNT162b2 vaccination}

(a-d) heatmaps revealing average fold changes of half maximal inhibitory concentrations (IC $\left.\mathrm{C}_{50}\right)$ normalized to $10^{7}$ viral RNA copies for serum infection-neutralization of EU1 and VoCs alpha, beta and gamma between the respective time points. pre - prior to first vaccination; \#1 - first vaccination; \#2 - second vaccination; \#3 - third vaccination; w2 - two weeks after respective vaccination; $\mathrm{m} 4-4$ months after vaccination; $\mathrm{m} 7$ - 7 months after vaccination. 
- Wratil et al: Superior immunity allows neutralization of all SARS-CoV-2 VoCs -

\section{Extended Data Figure 6}

a

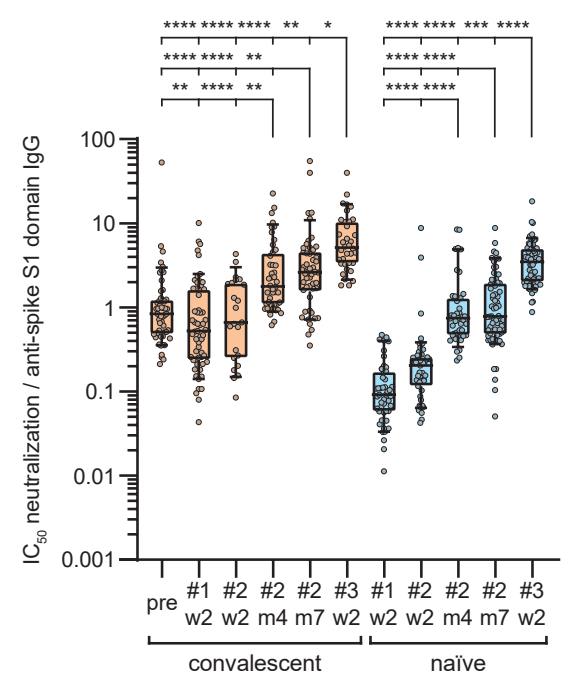

d

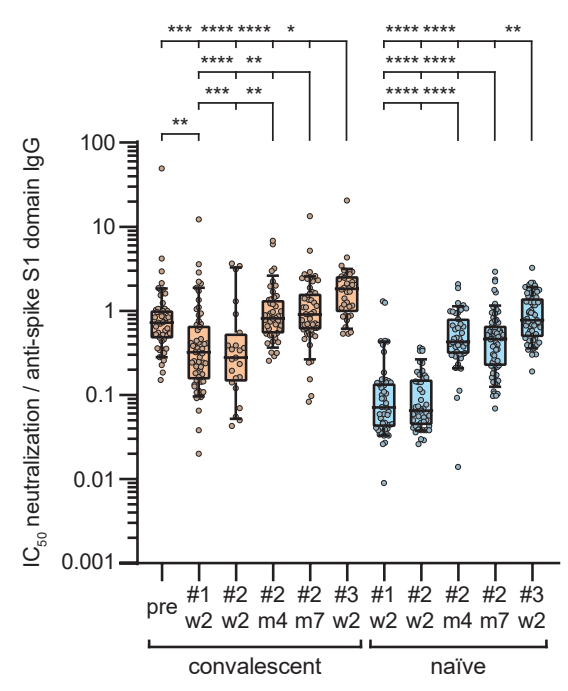

b

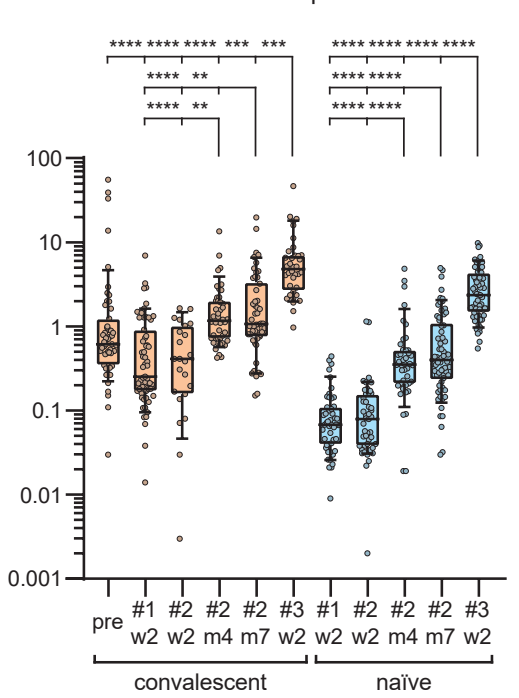

e

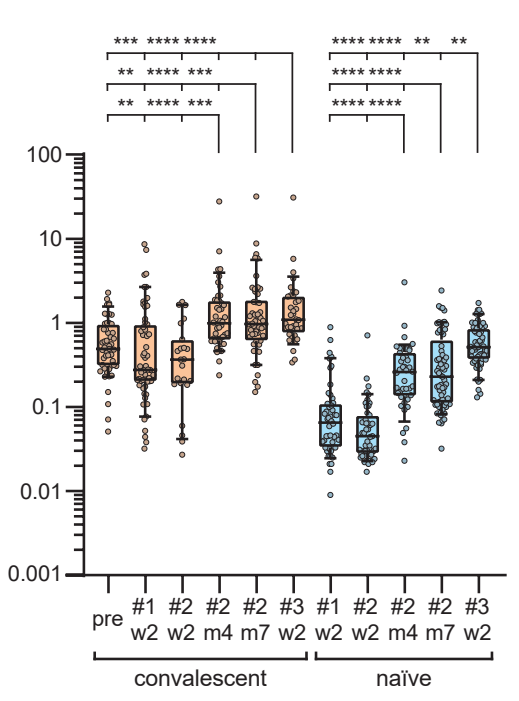

c

Beta

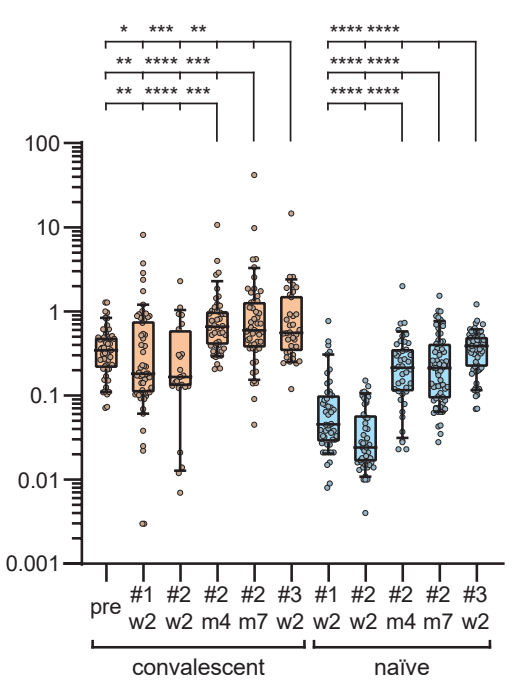

$\square$ convalescent

$\square$ naïve

f

Omicron

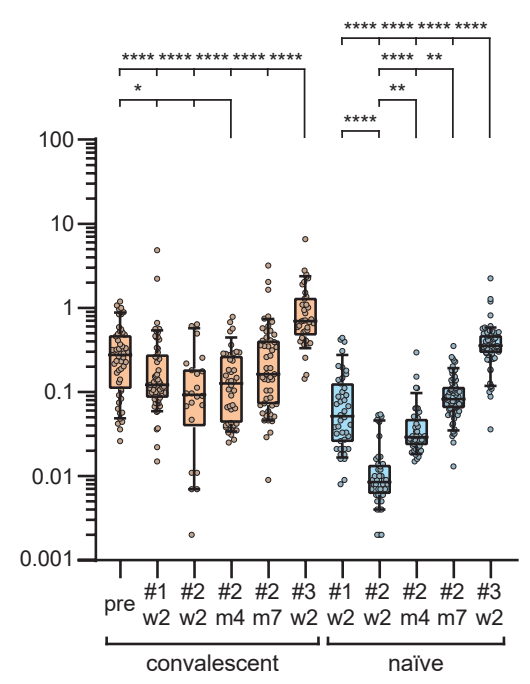




\section{Extended Data Figure 6. Longitudinal comparison of the ratios between infection neutralization activities against SARS-CoV-2 variants and anti-SARS-CoV-2 antibodies in naïve individuals and convalescents following BNT162b2 vaccination}

The ratios between half maximal inhibitory concentrations $\left(\mathrm{IC}_{50}\right)$ normalized to $10^{7}$ viral RNA copies for serum infection-neutralization of SARS-CoV-2 variants and antibody responses to the S1 domain of SARS-CoV-2 spike antigen are shown as box plots with whiskers between the $10^{\text {th }}$ and $90^{\text {th }}$ percentile in SARS-CoV-2 convalescents (regardless of whether or not they received vaccination \#2, orange) and naïve participants (blue). (a-e) 263 sera from 62 convalescents, and 295 sera from 73 naïves; (f) 258 sera from 62 convalescents, and 278 sera from 73 naïves. Differences between time points were analyzed for their statistical significance using the KruskalWallis test with Dunn's multiple testing correction. Connecting lines indicate statistically significant differences between groups: ${ }^{*} p \leq 0.05,{ }^{* *} p \leq 0.01,{ }^{* \star *} p \leq 0.001,{ }^{\star * \star *} p \leq 0.0001$. If no connecting lines or asterisks are given, no significant differences were detected.

pre: prior to first vaccination; \#1 - first vaccination; \#2 - second vaccination; \#3 - third vaccination; w2 - two weeks after respective vaccination; m4 - 4 months after vaccination; m7 - 7 months after vaccination. 


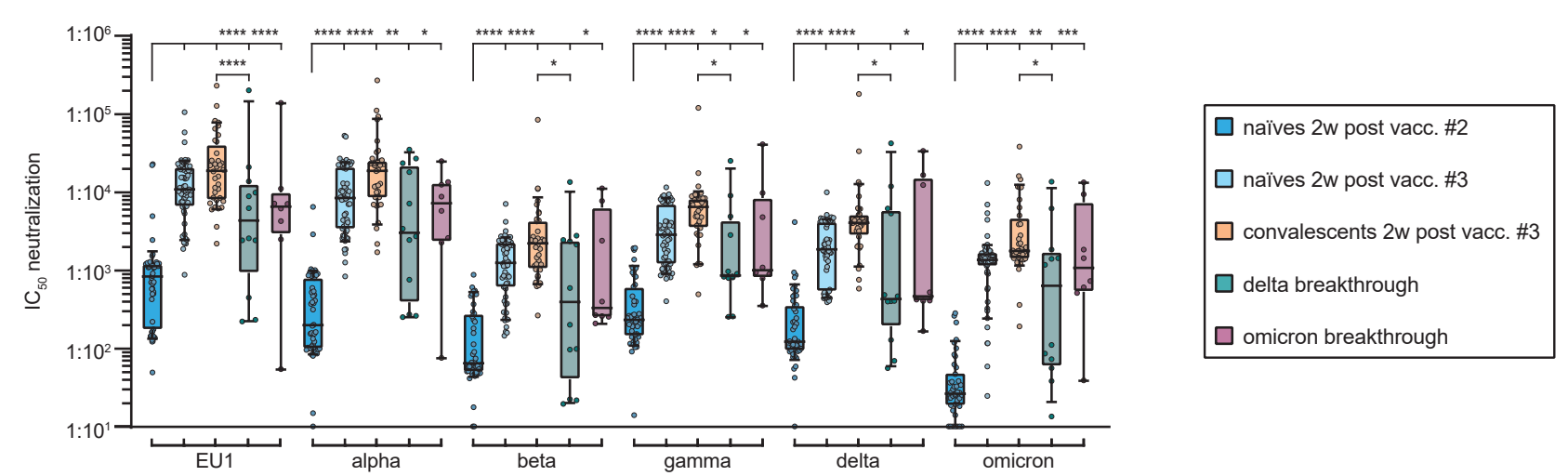




\section{Extended Data Figure 7. Comparison of infection neutralization activities for SARS-CoV-2 variants in twice BNT162b2 vaccinated COVID-19 patients after breakthrough infection with delta and omicron compared to naïve individuals and SARS-CoV-2 convalescents}

Half maximal inhibitory concentrations $\left(\mathrm{IC}_{50}\right)$ for serum infection-neutralization normalized to $10^{7}$ viral RNA copies of SARS-CoV-2 variants are shown as box plots with whiskers between the $10^{\text {th }}$ and $90^{\text {th }}$ percentile in 47 naïve participants (42 in case of omicron) 2 weeks after vaccination \#2 (dark blue), 59 naïve (light blue) and 36 convalescent participants 2 weeks after vaccination \#3, as well as 12 and 8 twice BNT162b2 vaccinated individuals on average 7 days after PCRconfirmed breakthrough infections with delta (green) or omicron (purple), respectively. Differences between groups were analyzed for their statistical significance using the Kruskal-Wallis test with Dunn's multiple testing correction. Connecting lines indicate statistically significant differences between groups: ${ }^{*} p \leq 0.05,{ }^{* *} p \leq 0.01$. If no connecting lines or asterisks are given, no significant differences were detected. 


\section{Supplementary Files}

This is a list of supplementary files associated with this preprint. Click to download.

- 220103WratilSternPrilleretalnrreportingsummary.pdf

- flatProtzerrs.pdf

- nreditorialpolicychecklistOKUP.pdf

- flatProtzerepc.pdf 